\title{
The Level-1 Trigger Barrel System of the ATLAS Experiment at CERN
}

\author{
F.Anulli ${ }^{1}$, G.Ciapetti ${ }^{1}$, D.De Pedis ${ }^{1}$, A.Di Girolamo ${ }^{1}$, C.Luci ${ }^{1}$, \\ A.Nisati $^{1}$, E.Pasqualucci ${ }^{1}$, F.Pastore ${ }^{1}$, E.Petrolo ${ }^{1}$, S.Rosati ${ }^{1}$, \\ F.Spila ${ }^{1}$, R.Vari ${ }^{1}$, S.Veneziano ${ }^{1}$, L.Zanello ${ }^{1}$, A.Aloisio ${ }^{2,3}$, \\ M.G.Alviggi ${ }^{2,3}$, M.Biglietti ${ }^{2,3}$, V.Canale ${ }^{2,3}$, L.Capasso ${ }^{2}$, \\ G.Carlino $^{2}$, F.Conventi ${ }^{2,4}$, R.DeAsmundis ${ }^{2}$, M.Della Pietra ${ }^{2,4}$, \\ D.Della Volpe ${ }^{2,3}$, R.Giordano ${ }^{2}$, P.Iengo ${ }^{2}$, V.Izzo ${ }^{2}$, S.Patricelli ${ }^{2,3}$, \\ G.Sekhniaidze $^{2}$, G.Aielli ${ }^{5}$, P.Camarri ${ }^{5}$, R.Cardarelli ${ }^{5}$, A.Di \\ Ciaccio $^{5}$, R.Di Nardo ${ }^{5}$, B.Liberti ${ }^{5}$, F.Marchese ${ }^{5}$, A.Salamon ${ }^{5}$, \\ R.Santonico ${ }^{5}$, M.Bianco ${ }^{6}$, G.Chiodini ${ }^{6}$, E.Gorini ${ }^{6}$, A.Guida ${ }^{6}$, \\ F.Grancagnolo ${ }^{6}$, R.Perrino ${ }^{6}$, M.Primavera ${ }^{6}$, S.Spagnolo ${ }^{6}$, \\ S.Antonelli $^{7}$, L.Bellagamba ${ }^{7}$, D.Boscherini ${ }^{7}$, A.Bruni ${ }^{7}$, \\ G.Bruni $^{7}$, M.Corradi ${ }^{7}$, P.Giusti ${ }^{7}$, G.Iacobucci ${ }^{7}$, A.Polini ${ }^{7}$,

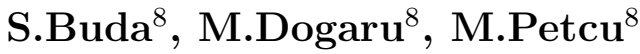 \\ ${ }^{1}$ Istituto Nazionale di Fisica Nucleare, Sezione di Roma, Universita di Roma La \\ Sapienza \\ ${ }^{2}$ Istituto Nazionale di Fisica Nucleare, Sezione di Napoli, Italy \\ ${ }^{3}$ Universita di Napoli Federico II, Italy \\ ${ }^{4}$ Universita di Napoli Parthenope, Italy \\ ${ }^{5}$ Istituto Nazionale di Fisica Nucleare, Sezione di Roma 2, Universita di Roma Tor \\ Vergata \\ ${ }^{6}$ Istituto Nazionale di Fisica Nucleare, Sezione di Lecce, Italy \\ ${ }^{7}$ Istituto Nazionale di Fisica Nucleare, Sezione di Bologna, Italy \\ 8 NIPNE HH, Bucarest Romania
}

\begin{abstract}
The ATLAS Level-1 Muon Barrel Trigger is one of the main elements of the first stage of event selection of the ATLAS experiment at the Large Hadron Collider. The challenge of the Level- 1 system is a reduction of the event rate from a collision rate of $40 \mathrm{MHz}$ by a factor $10^{3}$, using simple algorithms that can be executed in highly parallel custom electronics with a latency of order of $1 \mu \mathrm{s}$. The input stage of the Level-1 Muon consists of an array of processors receiving the full granularity of data from a dedicated detector (Resistive Plate Chambers in the Barrel). This first stage of the algorithm is performed directly on-detector, while the final stage is performed on boards mounted in the counting room, by the so-called off-detector electronics.

The trigger algorithm is executed within a fixed latency, its real-time output is the multiplicity of muon candidates passing a set of programmable $p_{T}$ thresholds, and their topological information. The detector system and the trigger electronics are designed to achieve a safe bunch-crossing identification. In order to optimize design effort and cost, the trigger system integrates also the readout of the detector, with its own requirements on time resolution and overall data bandwidth. We present the
\end{abstract}


detailed functional requirements of the Level-1 Muon Barrel system, its architecture, implementation and construction. 


\section{Introduction}

\subsection{Overview of the ATLAS Trigger System}

The ATLAS Trigger system is designed to perform event selection and data rate reduction, from $40 \mathrm{MHz}$ to $\mathrm{O}(100 \mathrm{~Hz})$ via three different trigger levels [1]. The trigger relies on the concept of trigger objects, which are identified and selected at first level. Reconstruction and selection are then progressively refined at higher levels, where all detectors data are accessible with full-granularity. Higher-level triggers rely on the topological information produced by Level-1, called Region-of-Interest (ROI), associated to each trigger object, to reduce the amount of data processed and transferred from detector to storage. Selection at first-level is based on inclusive high transverse momentum $\left(p_{T}\right)$ objects with low multiplicity, and energy sums sensitive to Standard Model and New Physics. The algorithms used are capable of a rate reduction down to an overall acceptance rate of about $100 \mathrm{kHz}$. A constraint of the system is the maximum fixed latency allowance of $2.5 \mu \mathrm{s}$, including processing time and propagation delays, coming from the need to store detector data on front-end electronics during the trigger decision.

To achieve these goals ATLAS has chosen a design based on dedicated hardware systems, including a Central Trigger Processor (CTP) which takes the final decision starting from information coming from the Calorimeter and Muon detectors. The Muon system, due to the spectrometer large volume and different detector choice for end-cap and barrel, is further subdivided in two parts, a Barrel part and and End-Cap part. A Muon to CTP Interface (MUCTPI) subsystem collects trigger objects from the Barrel and the Endcap subsystems and feeds the CTP. The ROI Builder is also part of Level-1, responsible for distributing ROI information to the next trigger level, Level-2. The Level1 trigger decision is fed-back to the front-end electronics and other readout components via the Timing, Trigger and Control System (TTC), based on a tree of optical and copper timing signal distribution boards and links.

\subsection{Overview of the ATLAS Muon Level-1 Trigger System}

The use of different detectors technologies, best adapted to precision measurement or trigger has been the ATLAS driving force in the choice of the detector. Monitored Drift Tubes (MDT) are used in offline reconstruction to provide precise sagitta measurement of muons bending in the spectrometer on the full detector acceptance. Only in the region $|\eta| \geq 2.0$ of the innermost muon station Cathode Strip Chambers are used.

Resistive Plate Chambers (RPC) are used as the Barrel Trigger detector in the region $|\eta| \leq 1.05$, while in the End-Cap Thin-Gap Chambers (TGC) are used [2].

1.2.1. Detector description Detectors providing signals to the Level-1 Muon Trigger electronics must provide fast information on muon tracks traversing the detector in an

environment of very high background. Main detector properties required for this purpose 
are: good time resolution for safe bunch-crossing identification; a spatial resolution allowing discrimination on muon transverse momenta and coarse tracking for input to higher-level triggers; second-coordinate measurement in the non-bending projection with a resolution of $\sim 10 \mathrm{~mm}$ per point, as required for the precision chambers measurement. The trigger detectors must provide acceptance in pseudo-rapidity out to $|\eta| \simeq 2.4$ and over the full $\phi$ range. Soft background in the cavern induces random hit rates in the trigger detectors in the range $20-60 \mathrm{~Hz} / \mathrm{cm}^{2}$ at high luminosity. This requires a coincidence of two or more planes along the triggering track. The RPC has a very good time resolution of $\sigma \sim 1.5 \mathrm{~ns}$, that allows bunch-crossing identification, and the spatial resolution can be tuned to the required need, by equipping the detector with $O(1 \mathrm{~cm})$ strips measuring bending and non-bending coordinates. Each RPC plane consists of two gas gaps, defined by resistive plates made of bakelite plates $\left(\rho=10^{10} \Omega \mathrm{cm}^{2}\right)$, separated by $2 \mathrm{~mm}$ high polycarbonate spacers placed every $10 \mathrm{~mm}$. The gas mixture is composed of $\mathrm{C}_{2} \mathrm{H}_{2} F_{4}(94.7 \%), I S O-C_{4} H_{10}(5 \%)$ and $S F_{6}(0.3 \%)$ and the chamber is operated in avalanche mode. The RPCs are organized in several modules and their dimensions have been chosen to match those of the corresponding MDT, to whom they are mechanically integrated. ATLAS physics benchmarks suggest two threshold regimes for muon triggers: the $h i g h-p_{T}$ regime $(10-40 \mathrm{GeV} / \mathrm{c}$ thresholds $)$ for heavy objects searches and a low $-p_{T}$ trigger $(4-10 \mathrm{GeV} / \mathrm{c})$ for b-physics studies. The trigger system architecture reflects this choice. In all the stations, RPC are composed of two units along both the azimuthal and beam direction. The so-called Middle Stations, at a radial distance of about $5 \mathrm{~m}$ from the interaction point contain two doublets of RPCs separated by a $\sim 0.7 \mathrm{~m}$ radial distance, called Confirm and Pivot doublets, while the so-called Outer Stations contain one doublet only, at $7 m$ radial distance [3]. Strip pitch has been optimized to minimize the number of channels and to make the measurement error due to detector segmentation negligible with respect to the overall smearing effect of $3 \mathrm{~cm}(\eta=0) \div 7 \mathrm{~cm}(\eta=1)$ for $p_{T}=6 \mathrm{GeV} / \mathrm{c}$ muons, and $3 \mathrm{~cm}(\eta=0) \div 6 \mathrm{~cm}(\eta=1)$ for $p_{T}=20 \mathrm{GeV} / \mathrm{c}$ muons [4]. Signals are induced on planes of orthogonal readout strips, four per doublet. To avoid dead areas between adjacent units, active zones of neighbouring RPCs are partially overlapped in $\eta$ by about one strip. There is a total of 1052 chambers covering a surface of about $3650 \mathrm{~m}^{2}$ providing $\simeq 350,000$ front-end signals.

1.2.2. The Barrel trigger geometry The ATLAS muon spectrometer in the barrel region is made of an air core toroid containing three measurement stations called respectively the Inner, Middle, Outer, respectively at about $3.5 \mathrm{~m}, 5 \mathrm{~m}, 7 \mathrm{~m}$ from the interaction point. It is subdivided in $\phi$ in eight so-called small and eight so called large Chamber Sectors. In the $\eta$ view it is divided in two parts, $\eta<0$ and $\eta>0$. From the trigger point of view, the barrel system is segmented in 64 sectors, in the $\phi$ projection 32 sectors per half-barrel. Each physical chamber in the Pivot plane defines two trigger regions in the $\eta-\phi$ plane, called PADs, belonging logically to two trigger sectors, as shown in picture 6. One PAD region has a granularity of $\Delta \eta \times \Delta \phi \simeq 0.2 \times 0.2$. A Region of 


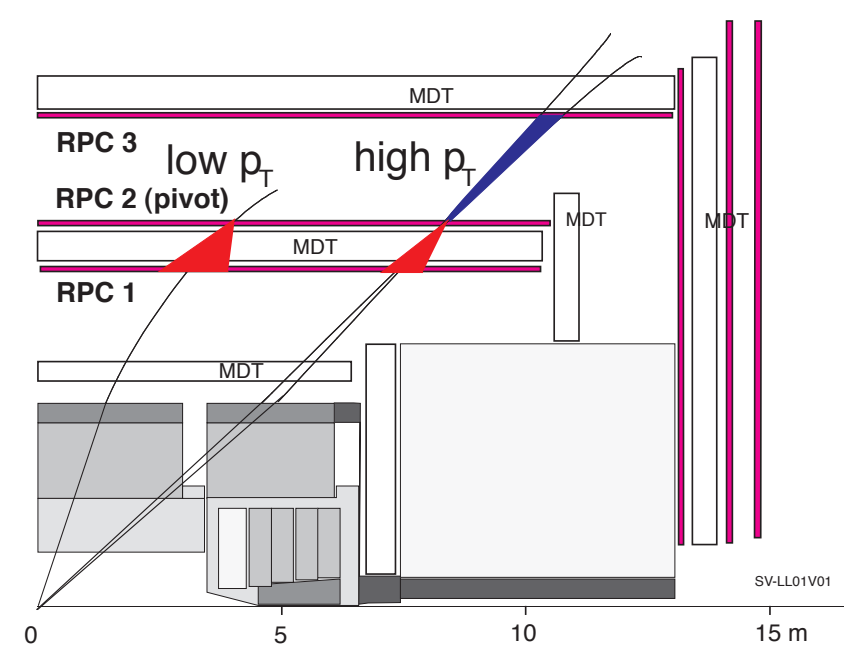

Figure 1. The barrel algorithm

Interest (ROI) within a PAD is defined by the logical overlap between areas covered by $\eta$ and $\phi$ Coincidence Matrices (CM), described later, giving a final granularity of $\Delta \eta \times \Delta \phi \simeq 0.1 \times 0.1$. The area processed by a CM has been chosen as a compromise on the maximum number of input channels to a single $\mathrm{CM}$ and the lowest transverse momentum threshold achievable given the detector strip pitch.

1.2.3. Overlap handling Concerning the trigger segmentation, a relevant issue for a trigger where candidate multiplicities are used is the overlap handling. Overlap between different parts of the apparatus cannot be avoided without loosing efficiency in uncovered regions. On the other hand overlap can cause double counting of muon candidates. The problem of overlaps inside a PAD region is solved locally by the PAD processing units that removes double counting of tracks between the four ROIs of the region. In addition, if it is found that a trigger was generated in a zone of overlap with another Pad region, the trigger is flagged as such, and any overlap will be solved at Sector level. Consequently each sector prevents double counting of triggers in the overlap region between two Pads, and flags triggers generated in regions of overlap between Sectors either belonging to the barrel or belonging to the endcap. This kind of overlap is easily solved in the MUCTPI, where information from all sectors is collected.

\subsection{The Barrel Trigger Algorithm}

The design of the Level-1 Muon Barrel has been driven by a simple concept (Figure 1). The trigger is done with three planes segmented in strips in the $\mathrm{r}-\mathrm{z}$ and $\mathrm{r}-\phi$ projections. Using as origin the nominal position of the p-p interaction region, the strip hit in the central plane of the Middle Station (RPC2) defines for low transverse momentum particles a coincidence window where to search for a correlated hit in the inner plane (RPC1) of the same Station (low $p_{T}$ algorithm). For higher momentum particles, then 
the strip hit in the middle plane defines also a new coincidence window where to search for a correlated hit in the outer plane (RPC3) of the Outer Station (high $p_{T}$ algorithm). The trigger is done on both bending and non-bending projections. The three planes are made of two detector layers, and the order of the coincidence logic (2-out-of-4, 3-out-of-4, 4-out-of-4 majorities) is defined to maximize the efficiency and to minimize the rate due to uncorrelated and correlated background. The algorithm is executed in parallel on a number of coincidence windows, each window calculated with an acceptance probability for $p_{T}$ greater than a given threshold. Smearing effects to the coincidence windows are due to fluctuations of energy loss in the calorimeter, length of the interaction region, multiple Coulomb scattering in the calorimeter and non-uniformity in the non bending projection of the magnetic field. The detector segmentation is chosen to make the error negligible compared to these effects [4]. Such an algorithm can be implemented using an array of dedicated processors, running in parallel and placed physically close to the data source, i.e. on-detector, and arranging the data processing in projective trigger towers.

\subsection{Input data}

The trigger system processes data using the full detector granularity of about 350,000 strips, sampled at a rate of $8 \times 40 \mathrm{MHz}$. This sampling period of $3.125 \mathrm{~ns}$ has been chosen as the internal system clock of the Coincidence Matrix Processors because it is comparable to the detector resolution, becoming the basic time unit in the digital delay lines used in the trigger part for the alignment of signal coincidences and in the readout part for hit time measurements. The total input aggregated bandwidth is $\sim 112 \mathrm{Tbit} / \mathrm{s}$.

\subsection{Output data}

The total throughput of the system on the trigger data path is $81.92 \mathrm{Gbit} / \mathrm{s}$ given by 64 trigger sector output producing a new result every $25 \mathrm{~ns}$. Each trigger word contains details of up to two muon candidates, their spatial position on the $\eta-\phi$ plane, the highest threshold passed by the candidates, overlap flags indicating if the candidates have been found in a region of overlap between trigger sectors, some timing information used to synchronize the system. Regarding the readout datapath, the system delivers to the data acquistion system details of detector hits, trigger hits, and intermediate trigger results at every Level-1 Accept, through 32 optical links running at $1.28 \mathrm{Gbit} / \mathrm{s}$.

\section{System description}

\subsection{System requirements and constraints}

The trigger algorithm defined in the previous chapters needs input data from all stations from a small region in the $\eta-\phi$ space. The obvious choice in the system architecture has been to organize data collection in trigger towers. This architecture is highly parallel 
and coupled with the need to minimize the number of data links has lead to the choice of putting most of the trigger electronics on-detector. Local processors called PADs contain all elements of the trigger pipeline needed to process data in a small detector region $(\Delta \eta \times \Delta \phi \simeq 0.2 \times 0.2)$, while electronics mounted in standard crates, sitting in the control room, takes care of processing data from a large part of the apparatus $(\Delta \eta \times \Delta \phi \simeq 1.0 \times 0.2)$. The main purpose of this electronics, called off-detector, is the counting of candidate multiplicities and the handling of overlap regions which might lead to double counting of single muon candidates.

The full trigger chain has been designed for robustness, capable to sustain high background rates and to handle regions of detector inefficiencies. The majority level of the time and spatial coincidence is programmable, and it has been foreseen to mask input channels with the granularity of a single strip. In order to further improve on robustness, it is also possible to include in the $l o w-p_{T}$ algorithm the outer detector plane. The improvements obtainable in presence of correlated background are well illustrated in [3], [5].

Other requirements for the trigger system are the bunch-crossing identification capability, necessary to an efficient reconstruction, and the data bandwidth reduction of all ATLAS detectors down to a sustainable value. These goals can be achieved by segmenting the detector in such a way that time-walk due to propagation delay along detector strips is much smaller that the machine crossing period (about $25 \mathrm{~ns}$ ) and by optimizing the electronics design.

A further requirement is the choice, for all ATLAS front-end electronics, of storing data before the level- 1 decision for a maximum time of $2.5 \mu \mathrm{s}$. This requirement leads to a maximum processing time of about $1 \mu \mathrm{s}$, considering that propagation delay along optical links and electronics connecting the level-1 processors to the front-end electronics takes a similar time, also about $1 \mu s$.

The expected radiation environment foreseen in the ATLAS cavern has been estimated with extensive simulations, giving an upper limit on the expected charged particles and neutron flux where level-1 electronics is mounted. Assuming a large safety factor of $O(50)$, due to uncertainties in the simulation, CMOS devices process spread and lot-to-lot variations, the expected total dose in the hottest region is 210 Gray in ten years, the expected neutron fluxes is $5.6 \times 10^{11}$, while charged particle flux, capable of producing Single-Event-Effects (SEE) is roughly a factor five smaller. All these values are very similar to the radiation levels expected in space, and similar care is necessary in the development of the system to mitigate the effects of irradiation.

Due to changing magnetic field along Monitored Drift Tube wires, to reach a contribution to the spatial resolution of $\mathrm{O}(10 \mu \mathrm{m})$ in the track measurement it becomes important to measure the second coordinate with a precision of about $1 \mathrm{~cm}$. For this reason the RPC detector has been segmented in strips also in the non-bending plane.

Considering all requirements described so far, the needs in time and spatial resolutions are respectively $\mathrm{O}(2-3 n s)$ in time and $\mathrm{O}(1 \mathrm{~cm})$ on the coordinate measurement. 


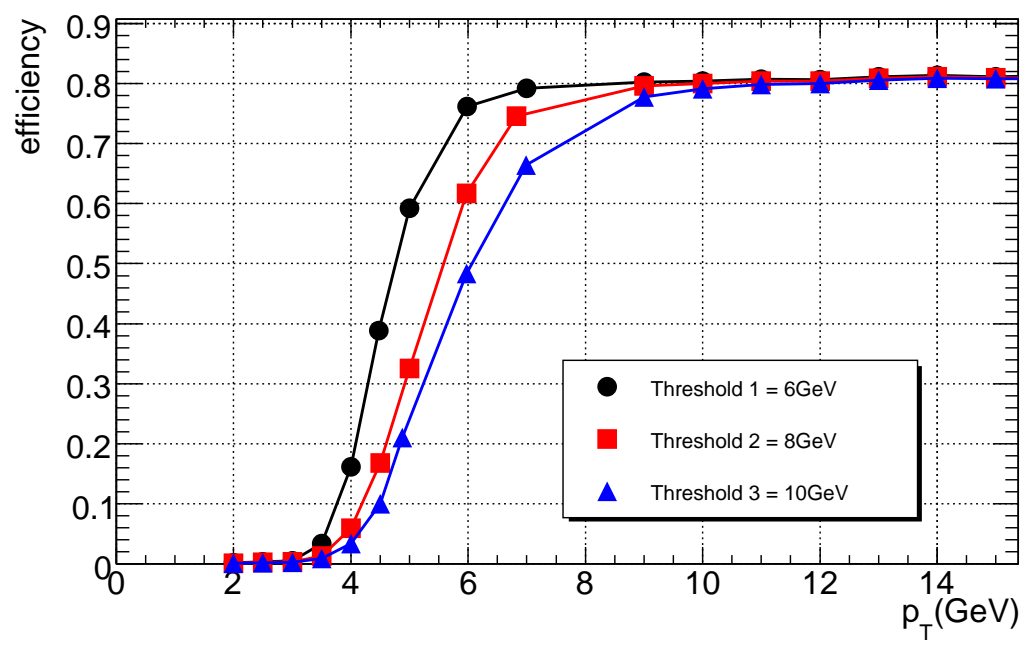

Figure 2. Low $p_{T}$ threshold efficiency curve.

2.1.1. The trigger efficiency and expected rates Efficiency curves as a function of $p_{T}$ have been produced using a detailed detector simulation of a large sample of single muon events $\left(10^{6}\right)$ with a wide $p_{T}$ range $(3-50 \mathrm{GeV} / \mathrm{c})$ [6]. The efficiency curves have been determined both for the $l o w-p_{T}$ and the $h i g h-p_{T}$ parts and are shown in figures 2 and 3 . The curves reach a plateau at about 0.82 and 0.78 respectively. This inefficiency is mainly due to reduced geometrical acceptance of some sectors of the barrel spectrometer. Two small sectors in the feet region in particular have a 0.05 loss of acceptance due to the presence of the structures supporting the magnet.

Also the level-1 trigger rates have been calculated for different machine luminosity scenarios, for single muons, from the convolution between the inclusive cross-sections for the main decay modes and the calculated trigger efficiency curves. The inclusive muon cross-sections at the LHC for decays of b anc c hadrons, top quark and $\mathrm{W} / \mathrm{Z}$ boson decays have been calculated using the Montecarlo program Pythia 6.13 [7], while the inflight decays of $\pi$ and $\mathrm{K}$ mesons have been calculated using the DPMJET Montecarlo program [8]. The cross-sections integrated in the kinematic region $\left(\eta<2.7 p_{T}>3 \mathrm{GeV} / \mathrm{c}\right)$, as a function of $p_{T}$ are shown in figure 4 .

At lower $p_{T}$ the $\pi / \mathrm{K}$ decays are the dominant source of muons while for $p_{T}>$ $8 \mathrm{GeV} / \mathrm{c}$ the cross-section is dominated by semileptonic decays of $\mathrm{b}$ and $\mathrm{c}$ hadrons.

Table 1 shows the estimated rates for the standard thresholds of $5-40 \mathrm{GeV} / \mathrm{c}$, for luminosities of $L=10^{31} \mathrm{~cm}^{-2} \mathrm{~s}^{-1}$ to $L=10^{34} \mathrm{~cm}^{-2} \mathrm{~s}^{-1}$.

2.1.2. The readout scheme The readout scheme foresees the combination of detector and trigger information in a single data fragment. This choice allows the development of a single device for all readout and trigger requirements thus reducing manpower needed for developments. The readout fragments contains raw RPC hit information with a time measurement bin of $1 / 8$ of the bunch crossing period, comparable to the detector 


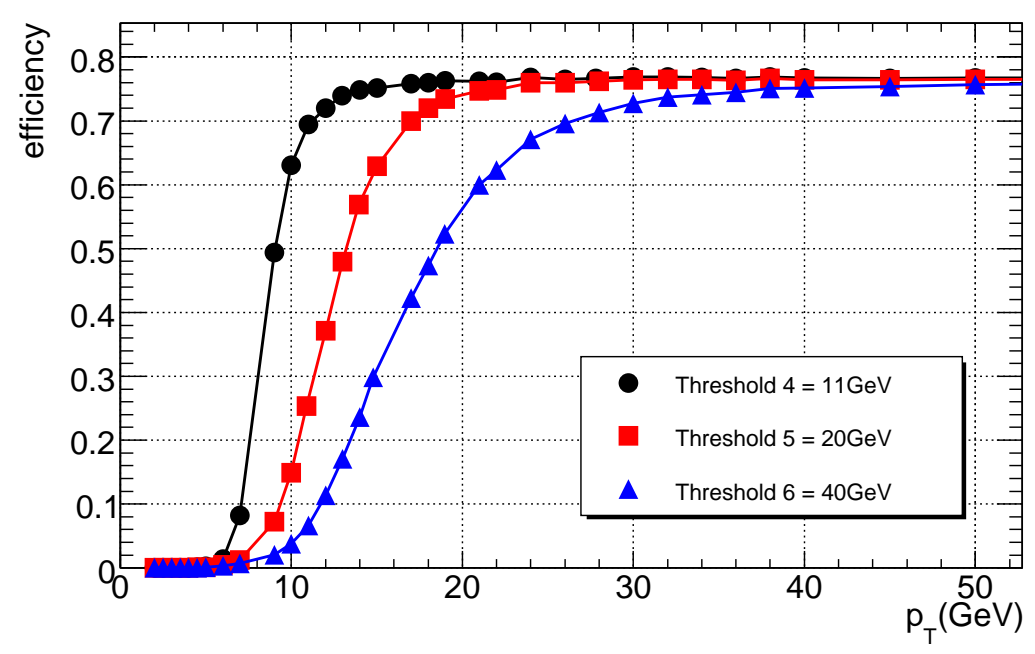

Figure 3. High $p_{T}$ threshold efficiency curve.

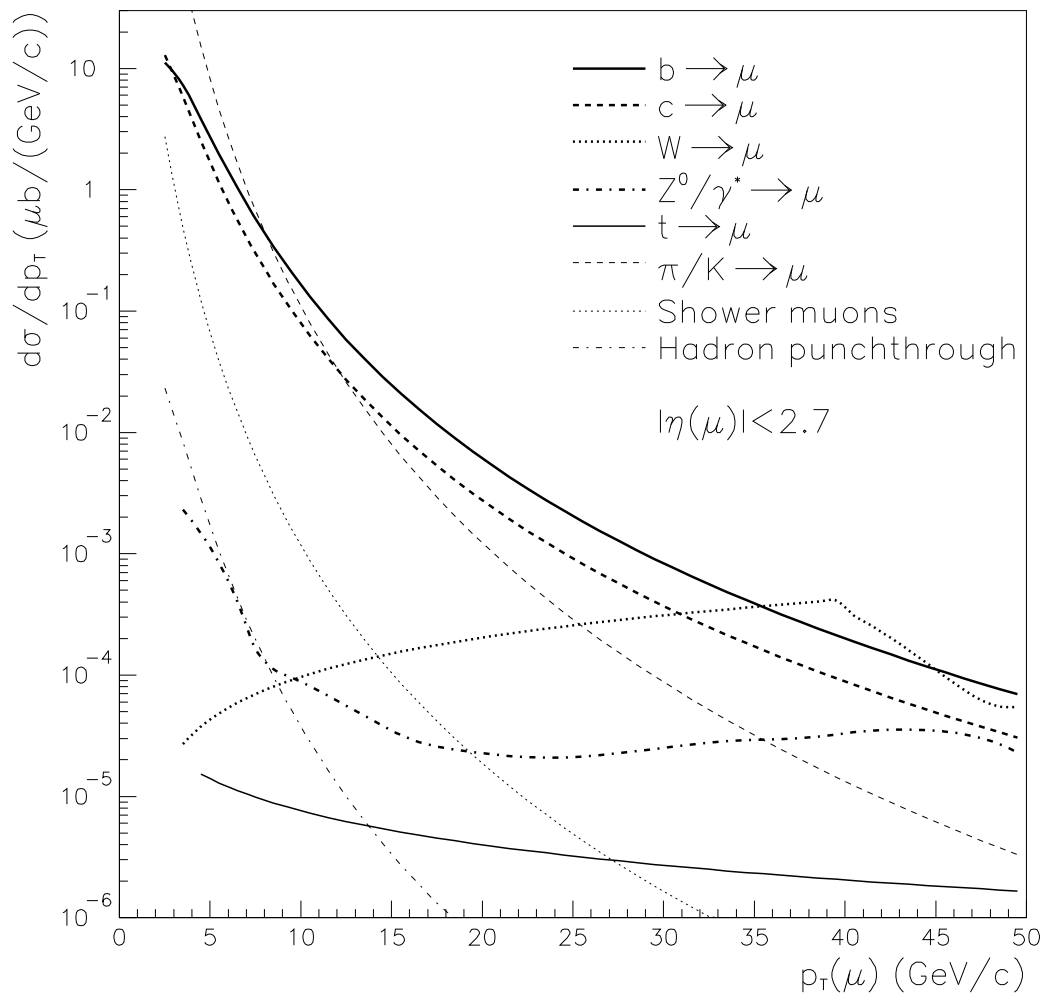

Figure 4. Muon differential cross-sections. 


\begin{tabular}{|c|c|c|c|c|}
\hline \multicolumn{5}{|c|}{ L1 muon trigger rates } \\
\hline $\mathrm{L}=10^{31}$ & \multicolumn{2}{|c|}{ "Cosmic" } & \multicolumn{2}{|c|}{$5 \mathrm{GeV} / \mathrm{c}$} \\
\hline $\mathrm{cm}^{-2} \mathrm{~s}^{-1}$ & Barrel $(\mathrm{Hz})$ & Endcaps $(\mathrm{Hz})$ & Barrel $(\mathrm{Hz})$ & Endcaps $(\mathrm{Hz})$ \\
\hline$\pi / K$ & 454 & 199 & 162 & 81 \\
\hline beauty & 85 & 74 & 54 & 53 \\
\hline charm & 124 & 104 & 76 & 73 \\
\hline top & 0 & 0 & 0 & 0 \\
\hline $\bar{W}$ & 0 & 0 & 0 & 0 \\
\hline TOTAL & 663 & 377 & 292 & 207 \\
\hline $\mathrm{L}=10^{33}$ & \multicolumn{2}{|c|}{$6 \mathrm{GeV} / \mathrm{c}$} & \multicolumn{2}{|c|}{$8 \mathrm{GeV} / \mathrm{c}$} \\
\hline $\mathrm{cm}^{-2} \mathrm{~s}^{-1}$ & Barrel $(\mathrm{Hz})$ & Endcaps $(\mathrm{Hz})$ & Barrel $(\mathrm{Hz})$ & Endcaps $(\mathrm{Hz})$ \\
\hline$\pi / K$ & 8600 & 5300 & 5200 & 2200 \\
\hline beauty & 4400 & 5100 & 3300 & 2900 \\
\hline charm & 6100 & 6900 & 4400 & 3800 \\
\hline top & 0 & 0 & 0 & 0 \\
\hline$W$ & 3 & 4 & 3 & 4 \\
\hline TOTAL & 19100 & 17300 & 12900 & 8900 \\
\hline $\mathrm{L}=10^{34}$ & \multicolumn{2}{|c|}{$20 \mathrm{GeV} / \mathrm{c}$} & \multicolumn{2}{|c|}{$40 \mathrm{GeV} / \mathrm{c}$} \\
\hline $\mathrm{cm}^{-2} \mathrm{~s}^{-1}$ & Barrel $(\mathrm{Hz})$ & Endcaps $(\mathrm{Hz})$ & Barrel $(\mathrm{Hz})$ & Endcaps $(\mathrm{Hz})$ \\
\hline$\pi / K$ & 1100 & 3800 & 470 & 1900 \\
\hline beauty & 2500 & 4000 & 1100 & 1300 \\
\hline charm & 2800 & 4700 & 1200 & 1400 \\
\hline top & 0 & 0 & 0 & 0 \\
\hline$W$ & 26 & 41 & 23 & 33 \\
\hline TOTAL & 6400 & 12500 & 2800 & 4600 \\
\hline
\end{tabular}

Table 1. Single muon trigger rates as obtained at L1, for different low and high $p_{T}$ thresholds, at $\mathrm{L}=10^{31} \mathrm{~cm}^{-2} \mathrm{~s}^{-1}, \mathrm{~L}=10^{33} \mathrm{~cm}^{-2} \mathrm{~s}^{-1}$ and $\mathrm{L}=10^{34} \mathrm{~cm}^{-2} \mathrm{~s}^{-1}$.

resolution of about $2 \mathrm{~ns}$, allowing a way of calibrating in time the trigger system, and to give a time-of-flight information to precision chambers with a better resolution than the bunch-crossing time. The same fragments contain also trigger hits information, for example the associated $p_{T}$ threshold firing the tower, if present. The data fragment contains also partial results of the trigger algorithm, as collected along the processing pipeline. A decision has also been taken regarding event building stages, so that event building occurs at every collection point along the chain, based on event tags as defined by the data acquisition community. The first collection point is the $H i g h-p_{T}$ ondetector processing elements (PADs), performing event building on a ratio of $8 / 1$, while second and third event building stages happen at the trigger sector level (Sector Logic, SL) and readout driver level (ROD), with ratios respectively of up to $8 / 1$ and $2 / 1$. 
Regarding the link choice, copper serial or parallel connections have been chosen for short connections, while for connections between cavern and the counting room, optical fibres have been chosen.

\subsection{Overview of the electronics system}

Signals from the RPC detector are amplified, shaped and discriminated inside the detector Faraday cage. The amplifier-shaper-discriminator boards, each containing eight front-end channels, are attached to the chamber and directly to RPC strips, embedded in the mechanical structure.

On-detector electronics includes also Splitters and PAD processors. They are attached on top of the detector inside metal boxes.

Regarding signal levels, front-end electronics delivers time-over-threshold signals on ECL levels. Since the rest of the system uses differential LVDS for all connections up to $10 \mathrm{~m}$ lengths, proper conversion is done where appropriate, namely at the input of Splitter and PAD elements.

Signals belonging to $\phi$ strips of the same chamber are wired-or before conversion and then sent to the Splitter and PAD boxes to reduce the channel count.

Digital time shaping of the front-end signals is done in the so-called Coincidence Matrix ASIC (CM), which performs almost all of the functions needed in the trigger and the readout system.

Each CM aligns in time the front-end signals, performs the time and spatial coincidence, and makes the $p_{T}$ cut on three different thresholds. The fullprogrammability of the geometrical coincidence has been done to avoid inefficiencies that may arise from non-ideal spectrometer geometry, and to minimize the effort on the electronics boards development. This device also contains the level-1 pipeline memory and derandomizing buffer. A detailed description of this chip is given later.

Signals from non-pivot detector doublets RPC1 and RPC3 are sent to so-called Splitter boards, whose main functionality is to collect front-end signals from one chamber and fan-out them to all adjacent PAD processors which need these data (maximum of two).

RPC signals from the first two detector doublets RPC1 and RPC2 are sent to a low $-p_{T}$ PAD processor containing $\eta$ and $\phi$ Coincidence Matrix boards (CM board). These dedicated CMs produce an output pattern containing the $l o w-p_{T}$ trigger results in the $\eta$ and $\phi$ projections independently. This pattern is sent to an high $-p_{T}$ PAD box via a bundle of LVDS links.

RPC signals from the RPC3 doublet, and the corresponding pattern result of the low $-p_{T} \mathrm{CMs}$, are also processed by CM boards. These boards, mounted inside the high $-p_{T}$ PAD box, contain the same Coincidence Matrix chip as used in the low $-p_{T}$ part, but they are programmed for the high $-p_{T}$ algorithm.

The cumulative data of four low and four high CM boards are finally processed in the so-called $h i g h-p_{T}$ PAD logic FPGA. This FPGA generates for each bunch crossing 


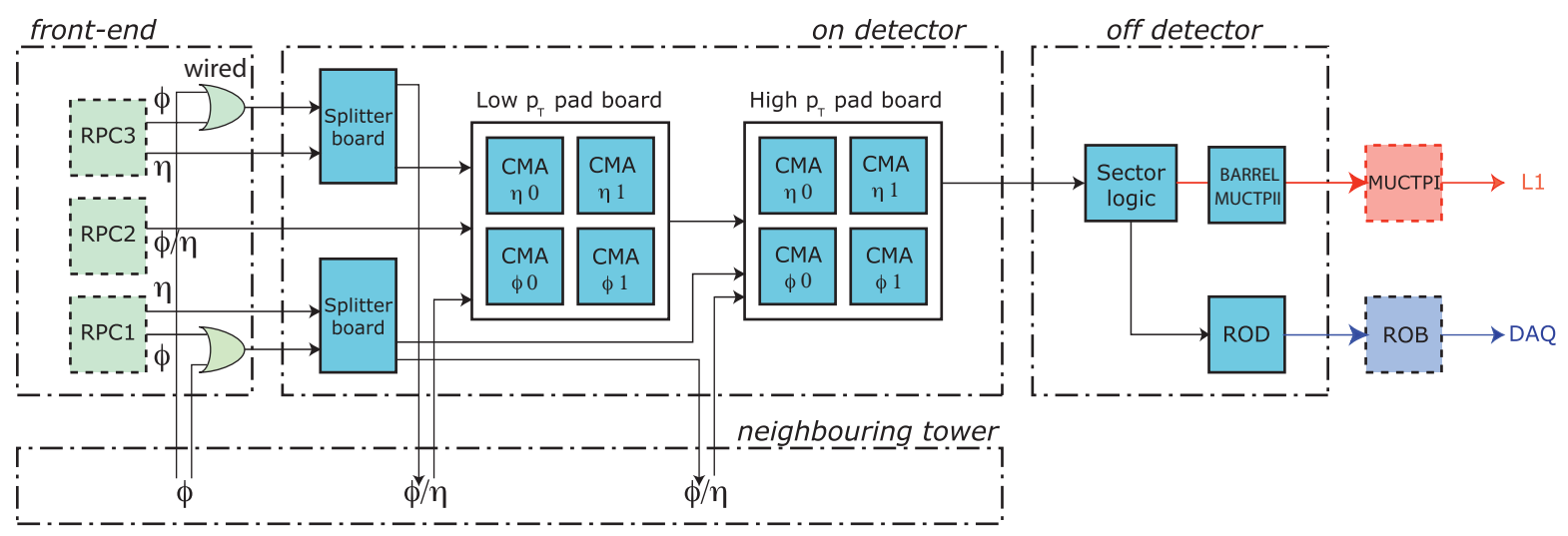

Figure 5. The barrel slice, showing the splitting of the system in front-end, ondetector and off-detector electronics. MUCTPI and ROB are respectively standard interfaces to the Central Trigger and the Data Acquisition System

a trigger result with the associated ROI information.

This information is transferred, synchronously at $40 \mathrm{MHz}$, to the corresponding Sector Logic module, that collects the overall result from all towers of a given sector (the barrel is sub-divided in 64 trigger sectors) [9], [10], [11].

Readout data follows the same route. All CM serial data outputs are sent along copper links from middle to outer stations, and inside the high $-p_{T}$ board to the PAD logic FPGA. This device processes readout data and outputs results.

Shipping of readout and trigger data is based on a time multiplexing mechanism where trigger data has higher priority. These multiplexed data are sent via an optical link to Sector Logic boards (SL). The link choice is based on a custom-made assembly based on Glink chipset.

Trigger and readout data are split only at the output of the SL board:

- readout data from two SL are collected by one Readout Driver Board (ROD), then shipped to DAQ;

- trigger data is sent via copper links from the SL to one input of the Muon to the Central Trigger Processor Interface (MUCTPI) via custom built interface boards (called Barrel MUCTPI Interface Board).

A simplified scheme of the trigger slice is described in Figure 5.

The off-detector electronics, housed in VME64x crates, uses a custom backplane bus to transmit readout and trigger data, along with all necessary flow control and timing signals. This backplane, called RODbus (Readout Driver bus), has a modularity of five slots, with slots dedicated respectively to ROD (central slot), Sector Logic (SL) (left-hand and right-hand slots) and MUCTPI interface (left-most and right-most slots). Details of this arrangement will follow later. 


\subsection{Segmentation}

Chamber types of varying sizes have been built to maximize hermeticity of the Muon Spectrometer. In this respect sizes vary from $1110 \mathrm{~mm} \times 4850 \mathrm{~mm}$ for largest chambers to $320 \mathrm{~mm} \times 530 \mathrm{~mm}$ for chambers closing gaps of magnet elements.

Being based on such a different chamber sizes, optimization of the electronics modularity has been a trade-off between number of strips per chamber, their pitch and lenght. The adopted solution has a reasonable number of readout channels,giving a reasonable Bunch Crossing identification capability, spatial and time resolutions and fanout. Strip pitches have beeen chosen to be $\simeq 3-4 \mathrm{~cm}$ and roughly projective when moving from middle to outer chambers. Strip lenghts vary within the ranges of $530-2425 \mathrm{~mm}$ for the eta strips, while the phi range is $480-1200 \mathrm{~mm}$. Concerning the front-end modularity, the basic segmentation is 8 channels, with normally two sets of 32 strips received per RPC doublet.

This modularity has been kept in the design of the electronics, by using 32-signal inputs per connector per processing element in the pivot plane of the $\eta$ view. Concerning the $\phi$ view, 32-channel inputs are required by the pivot plane, while 40 inputs are required per processing element in the confirm plane, so 32 and 40-input connectors have been used accordingly. It is important to note here that chamber sizes are not projective, and a trigger tower processes a variable number of front-end signals from the confirm plane. The choice done has been to keep 32-channel inputs for the $\phi$ pivot plane and keep confirm $\eta$ signals arranged in groups of four per cable (on RJ45 connectors). This last choice gives us a significant flexibility in arranging the inputs to CMs.

It is important to remember that to reduce the total number of channels in $\phi$, all strips belonging to a chamber being part of a tower are wired-OR if belonging to the same chamber, while up to a three-fold logical-OR is done on signals coming from adjacent chambers.

Since cable lengths are different for different towers, a detailed Montecarlo program has been used to define all cabling, to build a database then used for the actual cabling of the detector.

\subsection{The Splitter Board}

The Splitter box main requirement is to receive ECL-like signal from all front-end channels coming from confirm planes, translate into differential LVDS and fanout to processing unit. The receiving circuit carries also the front-end bias voltage.

Care has been taken to guarantee that maximum skew between channels is well below 1 ns, so that propagation delay on the boards does not deteriorate the detector time resolution. Simulation has been used to make sure that no need for a fan-out greater than two is required throughout the detector, in this case all Splitters are built using the same circuit layout. They are built out of a mainboard containing all voltage regulators and sensors, while for the $\phi$ and $\eta$ views different piggy boards are mounted. The modularity of the piggy boards has been chosen to match front-end, 32 inputs for 


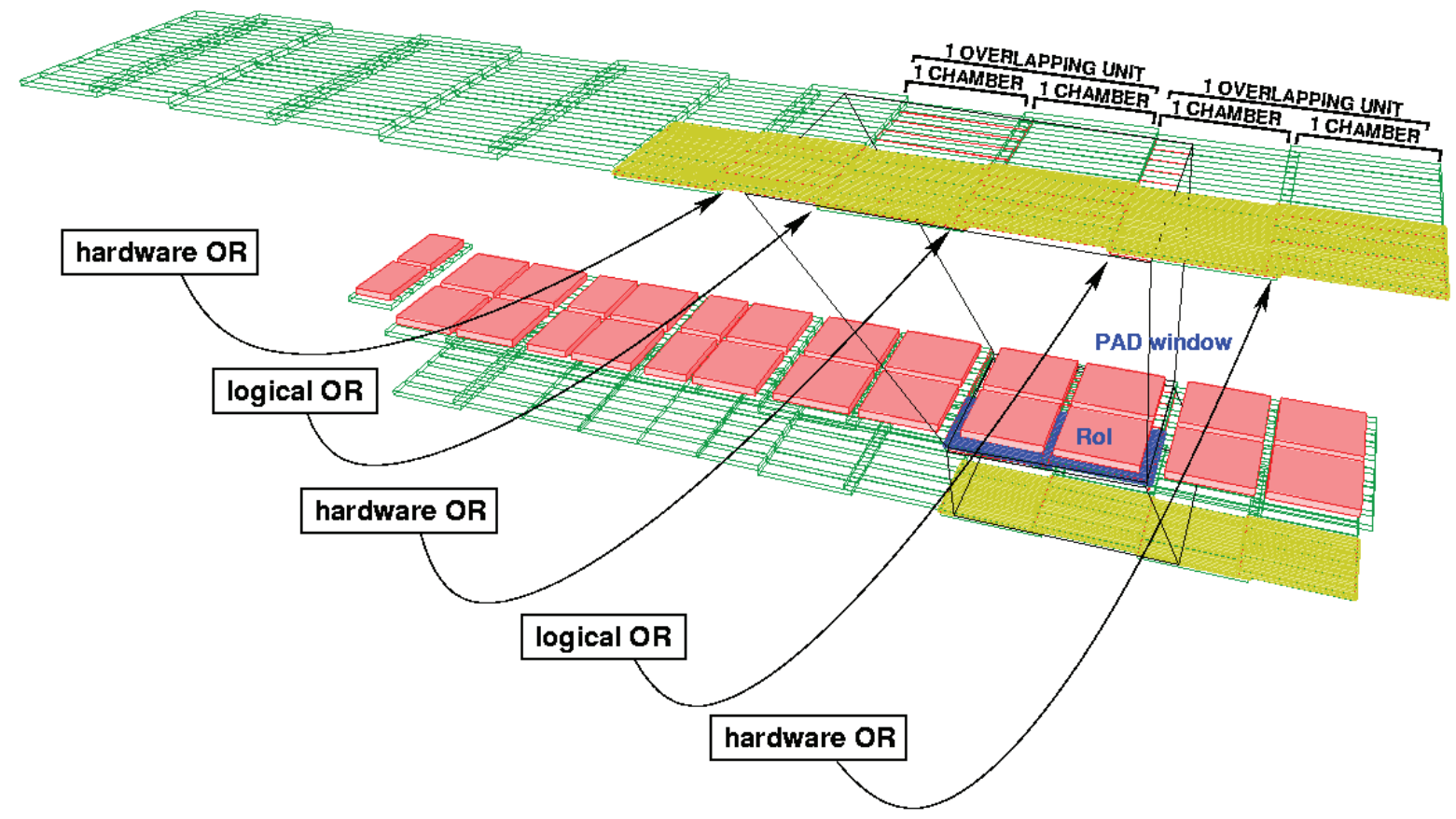

Figure 6. trigger segmentation in phi, in green are shown the various detector elements, in yellow all phi strips belonging to a tower are shown, where wired-OR and logical-OR is performed. In blue a PAD region is defined along with the PAD window reaching the inner and outer confirm planes.In red RoIs are shown.

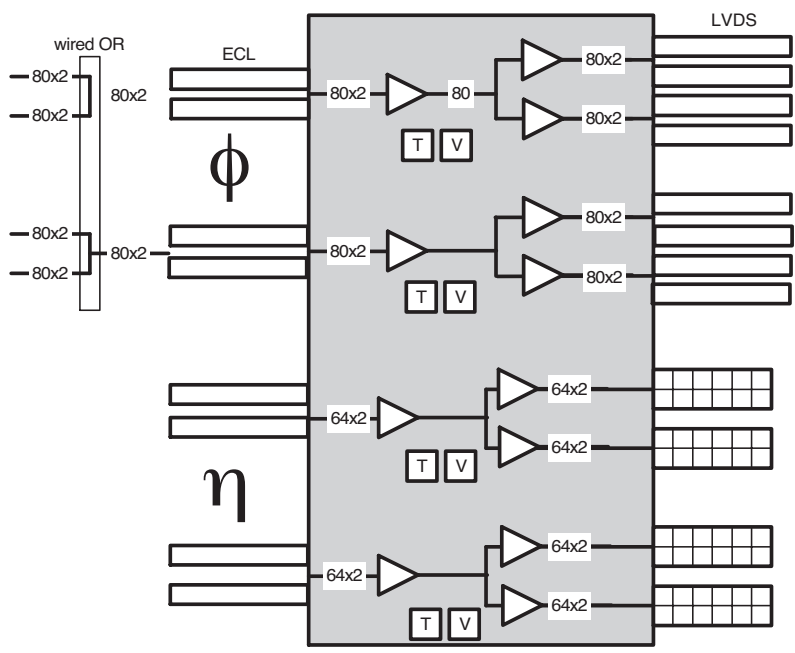

Figure 7. schematic diagram of a Splitter box. Granularity of the $\eta$ ouputs is 4channel 


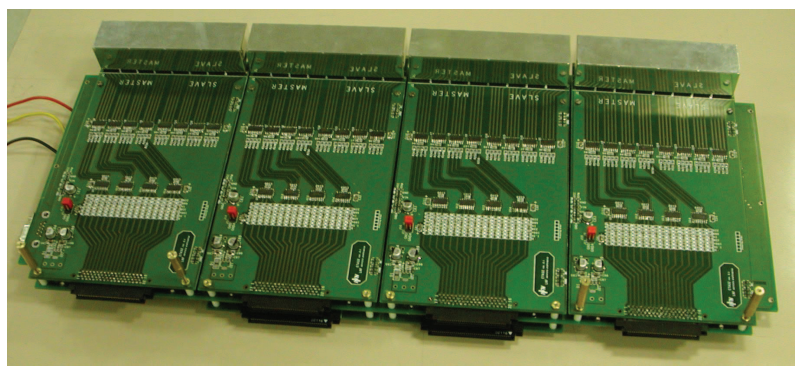

Figure 8. Picture of a Splitter circuit, as seen from the $\eta$ side, out of its encasing

$\eta$ strips and 40 for $\phi$ inputs. Input connectors are able to accept ribbon twisted cables and output connectors have been chosen to satisfy the necessity to implement tower projectivity by properly connecting Splitter outputs to PAD inputs. A granularity of 4 output channels per cable has been chosen on $\eta$ outputs, after Monte Carlo studies of all detector towers, while the granularity in $\phi$ is 40 , same as the inputs. Temperatures and voltages are monitored by a neighbouring PAD via an $\mathrm{I} 2 \mathrm{C}$ bus.

\subsection{The coincidence matrix ASIC}

Figure 9 shows the block diagram of the coincidence-matrix chip [25], [23], [12]. The chip receives the $40 \mathrm{MHz}$ machine clock and through a Delay Locked Loop (DLL) system, it generates a $320 \mathrm{MHz}$ internal clock that synchronizes all the pipeline operations inside the chip and a $160 \mathrm{MHz}$ clock used for all readout operations. The first block at the input of the chip is an edge detector and dead-time circuit. This block detects the rising edge of the arriving signals and, for each detected signal, sets a programmable dead time, of the order of one hundred nanoseconds, to avoid the arrival of extra pulses in the same RPC channel.

The second block allows for the possibility of masking RPC noisy channels. The third input block is a programmable-depth pipeline that is very important for setting up the timing of the system. Since the cables that bring the signals to the chip have different lengths, corresponding to different paths, this block allows for timing adjustment of the different groups of signals, in such a way that their coincidence is in time. The pipeline can be programmed in groups of eight RPC signals, with a minimum step corresponding to the period of the $320 \mathrm{MHz}$ internal clock (3.125ns). The RPC front-end takes care of aligning in time a group of eight signals, with a maximum spread of $1 \mathrm{~ns}$.

At the output of the programmable depth pipeline, the signals follow two different paths: one goes to the read-out part and one to the trigger part. In the trigger part, the first block is the programmable mask to 1 and pulse width setting circuit. This block allows for masking to " 1 " unused inputs to the matrix and for the digital shaping of the signal before making the trigger coincidence.

Digital shaping is necessary since the signals must be made as short as possible before the coincidence, to minimize the rate of fake triggers due to low-energy background particles in the ATLAS cavern (uncorrelated noise). This block allows 


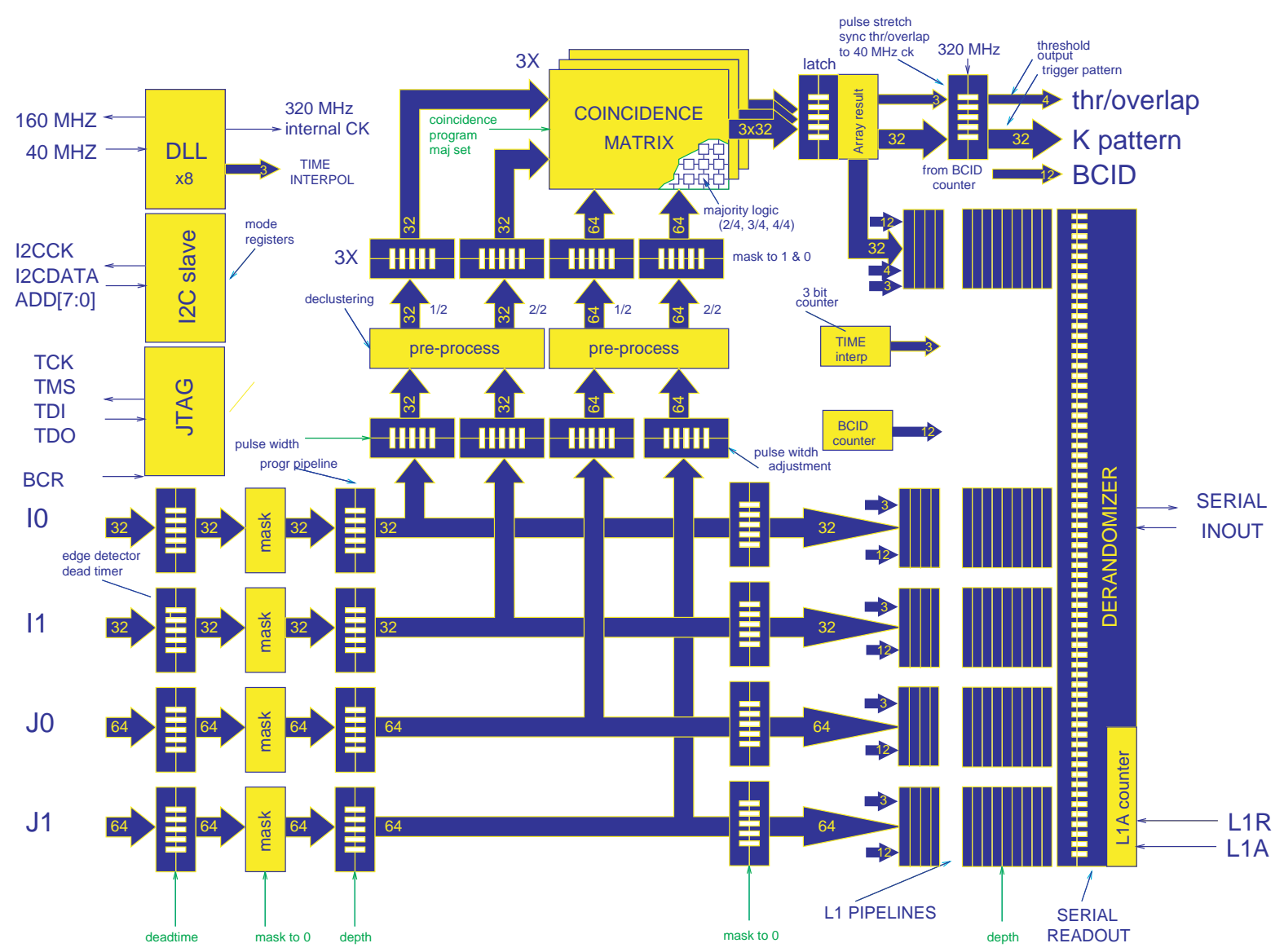

Figure 9. CM block diagram

for a programmable digital shaping of the signals, adjusting the duration in steps of $\simeq 3.125 \mathrm{~ns}$, corresponding to the internal clock period.

The next block is the pre-processing block. Here the declustering of the signals and the $1 / 2$ and 2/2 majority logic are performed. The RPC detector has an intrinsic cluster size of about 1.5 strips and a declustering algorithm is needed to define the cluster center.

The dimension of the matrix is $32 \times 64$. In the low-pT trigger, the signals coming from the RPC2 doublet are connected to the 32 I0 and 32 I1 inputs, and the signals coming from the RPC1 doublet are connected to the $64 \mathrm{~J} 1$ and $64 \mathrm{~J} 2$ inputs. In the case of the high-pT trigger the I0 inputs are used to connect the low-pT trigger result (the I1 inputs are not used), while the J0 and J1 inputs are both used to connect the high-pT RPC doublet.

The coincidence matrix has three times 32x64 coincidence cells, since the trigger must be performed on three different pT thresholds simultaneously. The cells have the possibility to be programmed at will, through a dedicated serial line, to perform $2 / 4,3 / 4$, or 4/4-majority logic, and to be set according to the required cut on the pT threshold.

The output of the coincidence matrix is sent to the readout part and to the trigger 
output part of the chip. Before producing the trigger output, the trigger pattern is synchronised back to the $40 \mathrm{MHz}$ clock. Typical latencies, when input pipelines are shortest, are about 75 ns to produce a trigger output. The 32-bit pattern contains the RPC2 doublet pattern that has generated the trigger and the associated coincidence timing. The trigger result (total of 7 bits) is produced synchronously with the $40 \mathrm{MHz}$ clock. Two bits are used for coding the highest $\mathrm{pT}$ threshold that was validated by the trigger (in case of no trigger both bits are zero), and two bits are used as a flag to indicate if the trigger occurred in a possible overlap zone (left or right) within the Pad region. The remaining three bits contain the low order bits of the Bunch Crossing Identification (BCID) number.

The main element in the read-out part of the chip is the level-1 pipeline memory. This memory stores the information for a time corresponding to the ATLAS level-1 trigger latency, which is $2.5 \mu \mathrm{s}$, including some contingency. The pipeline is implemented with a BCID-tagged and time-tagged set of FIFOs. The level-1 latency memory is subdivided in seven blocks: six blocks contain the information from the RPC doublets; one block contains the trigger array output result; each block contains also the 12-bit BCID counter and a 3-bit time interpolator value for a total of 47 bit words. This counter measures the phase of arrival of a frontend signal with respect to the external $40 \mathrm{MHz}$ clock with an LSB of one eight of the bunch crossing period and tells also to the read-out at which moment, within the bunch, the trigger occurred. This information is needed for the timing calibration of the system.

For events selected by the LVL1 trigger, data from the level-1 latency memory are transferred to the derandomizing memory from where they are read out serially.

The ASIC has been implemented in a 6-metal $0.18 \mu \mathrm{m}$ UMC process, with about 530kgates (about two million transistors) on an area of about $23 \mathrm{~mm}^{2}$ and since then it has been tested in various testbeams. Regarding testability, 32 internal logic scan chains have been implemented and used in production for acceptance tests (97\% coverage), and a full JTAG Boundary scan is available for tests after mounting.

The output bitstream can flow at a programmable speed of $10-80 \mathrm{Mbit} / \mathrm{s}$ in the DSlink format. The ASIC's (190) registers are fully programmable via the I2C interface. Most relevant registers, which may cause heavy loss of data in case of a Single Event Upset, are designed with redundancy in mind. They are triplicated and the output value is the one present in at least two of the register copies. Automatic recovery of the error is done on the triple redundancy registers and an SEU flagging bit is present to complete the system. All other registers only contain a parity bit and in case of a SEU a flag is raised. In this case the reprogramming of the ASIC internal register will restore the original value.

\subsection{The Processor Box (PAD)}

The PAD processor is made of a number of elements that are explained as follows:

- Motherboard. This board carries all processing units and their interconnections 


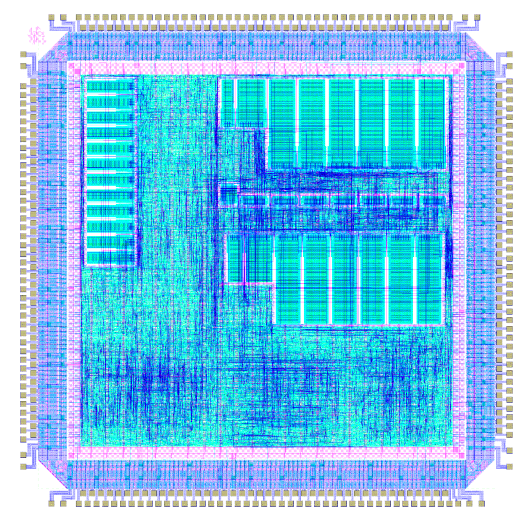

Figure 10. CM layout. On the upper left part of the die the input part is placed and is possible to see the input FIFO's implementing the delay line. On the upper right the readout processing is done and is possible to recognize the latency and output memories. On the lower part of the die the three coincidence matrices are implemented

and in case of the outer chambers also performs signal processing. It performs a logical-OR of signal inputs coming from one layer of $\phi$ strips of signals belonging to adjacent chambers.

It contains an FPGA (Xilinx XCV200), called PAD logic, which is programmed as the second readout and trigger processing stage, right after the CM ASICs. Delay chips are able also to fine tune internal phases of clock and other timing signals. Other services are present on the processor, like the possibility to store trigger configurations over local flash memories. In this case upon a configure command, delivered via CANbus, the local microcontroller is able to configure and check that the configuration has been executed with success. Another service, that has been used only before chamber installation, gives the possibility of delivering test pulses on response to specific TTC broadcast commands. It contains voltage regulators to differentiate voltage domains belonging to all piggy board circuits. Temperature sensors are also present to monitor local temperatures close to hottest components.

- Coincidence Matrix Board. Four are present, each board carries one CM ASIC and all input circuitry. The input part complexity resides in the way front-end signals connect to it. As already said a detailed Montecarlo has been used to produce the trigger connection at the input of this board and many thousands of cables have been produced of different lenghts in order to be able to keep the system in synchronism. Four variants have been built to be able to receive $\eta$ or $\phi$ inputs, and either LVDS or ECL translators (ECL from the front-end or LVDS receivers if inputs are coming from confirm planes or from the Low-Pt outputs).

- ORJ1-board. This board receives front-end signals and performs a logical OR of signals belonging to the second (confirm) layer of adjacent chambers, as required by the pointing geometry and tower definition.

- ELMB. This element is a CANbus controller, based on an ATMEL device (ATMEGA128), able to access all programmable elements of the PAD [13]. Three 


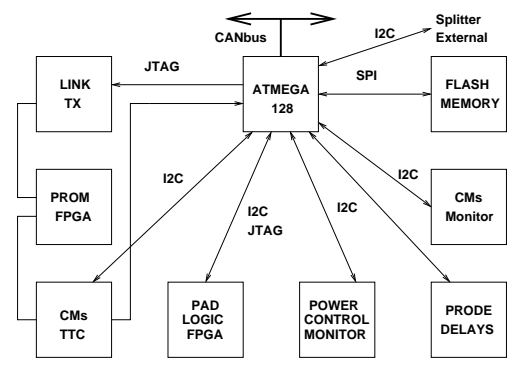

Figure 11. PAD control buses. I2C buses control most of the system, while JTAG is used mainly for the local FPGA. An SPI protocol is also implemented to access the external flash memory

I2C buses access IO registers, voltage and temperature regulators and ASICs, while a fourth $\mathrm{I} 2 \mathrm{C}$ bus is used to access one neighbouring splitter box. A JTAG bus is available to program the motherboard FPGA remotely.

- TTCRq. This is a standard Trigger and Timing receiver board used throughout the experiment. Its clock output and timing signals are reference to the whole processor.

- Optical link TX. The Optical link board is a custom board called GLINK, containing one HDMP1032 serializer and optical transceiver. The board is able to deliver a 17-bit word at $40 \mathrm{MHz}$.

2.6.1. The Low $-p_{T}$ and the $H i g h-p_{T} P A D S$ The two processor types share the same board layout but differ in the direction of some data paths and programmability of the system. In particular, as can be found in figures 12 and 13, the low $P_{t}$ board does not contain a TX mezzanine, all readout and trigger signals from low to high stations travel on LVDS differential connections. Data processing of each trigger tower, containing the low and the high $p_{T}$ parts, is done on the high $p_{T}$ PAD. The pipeline and readout stage past the CM ASICs is performed on the high $p_{T}$ processor, in the FPGA which we call PAD logic.

2.6.2. The PAD logic. The PAD logic firmware can be divided into two main parts:

- Readout part. This part takes care of receiving each of the eight CM serial inputs and deserialize into 16-bit words. Upon event request via a L1A, all internal FIFOs (up to 8) are scanned for data. If data belonging to the expected event number are present the CM fragment is shipped to an output FIFO and then to the optical TX. This output FIFO sends data to the corresponding Sector Logic board as soon as data are present. Since no XON-XOFF mechanism is available, the system must rely on a BUSY signal that must be propagated up to the ATLAS Central Processor. At this stage of the event building each PAD fragment contains eight $\mathrm{CM}$ fragments, each fragment containing the L1ID and BCID values that can be checked against the ROD value. 


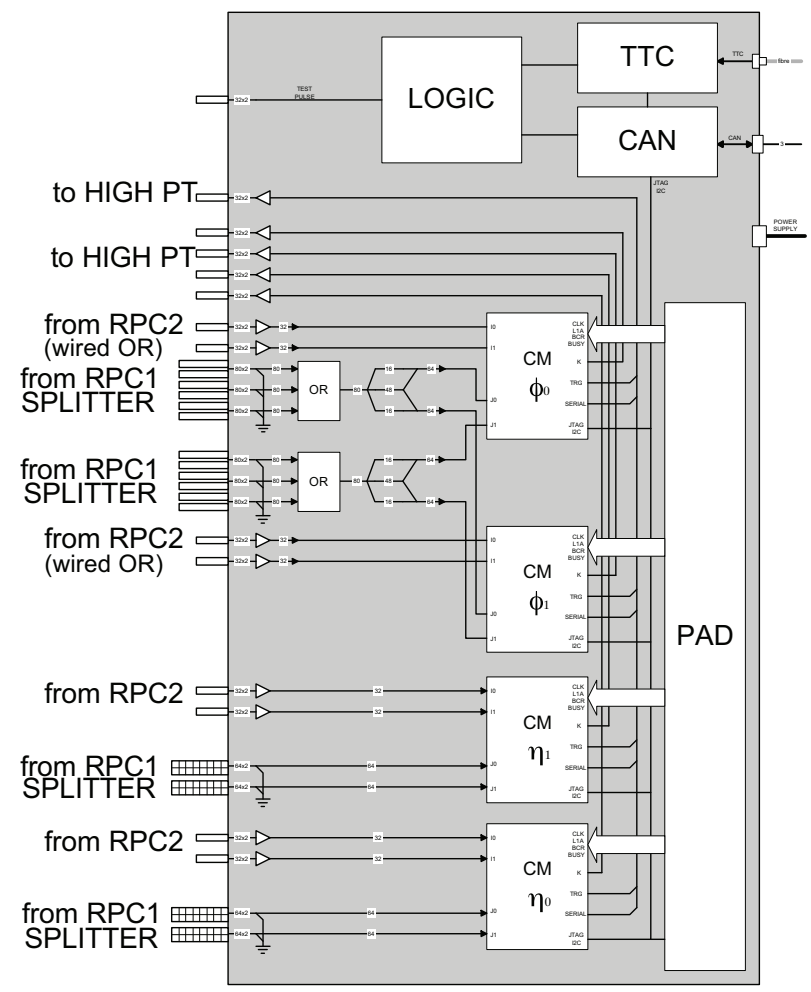

Figure 12. PAD low layout. The four CM's collect front-end signal (from RPC) and output the readout and trigger data (to HIGH PT) over external LVDSs buses. TTC and CAN are used for initializing and timing of the processor

- Trigger part. This part receives as input from each of the eight CMs values for Pt threshold, ROI, 3-bit BCID number and overlap flags. The input pipeline is able to process data with a latency of four BC's and find the highest Pt candidate in the tower, assigning it to a specific RoI. If more than one candidate is present, only the one with highest $\mathrm{Pt}$ value is sent to the optical link.

Readout and trigger data are time-multiplexed by the optical link logic present in the FPGA, by assuring that any trigger will be sent with a fixed latency, giving less priority to data words over trigger words. Other signals like busy status of the processor, are sent as special trigger words which produce no candidates. For further details see references [14], [15], [16].

\subsection{The optical interconnection}

The Level-1 (L1) Muon Barrel Trigger transmits data serially on a custom designed optical link. The TX and RX nodes (Fig. 15) implement a unidirectional optical link on multimode fibre driven by a 850nm Vertical Cavity Surface Emitting Laser (VCSEL), with a reach of $500 \mathrm{~m}$. The TX nodes plug into the L1 Muon Barrel Trigger processors resident on the RPC detectors, while the RX nodes are hosted on VME carrier boards in the counting room. The RX has a dual layout, with two independent receiver units. This allows concentrating on the VME board up to 8 L1 Trigger Processors outputs. In 


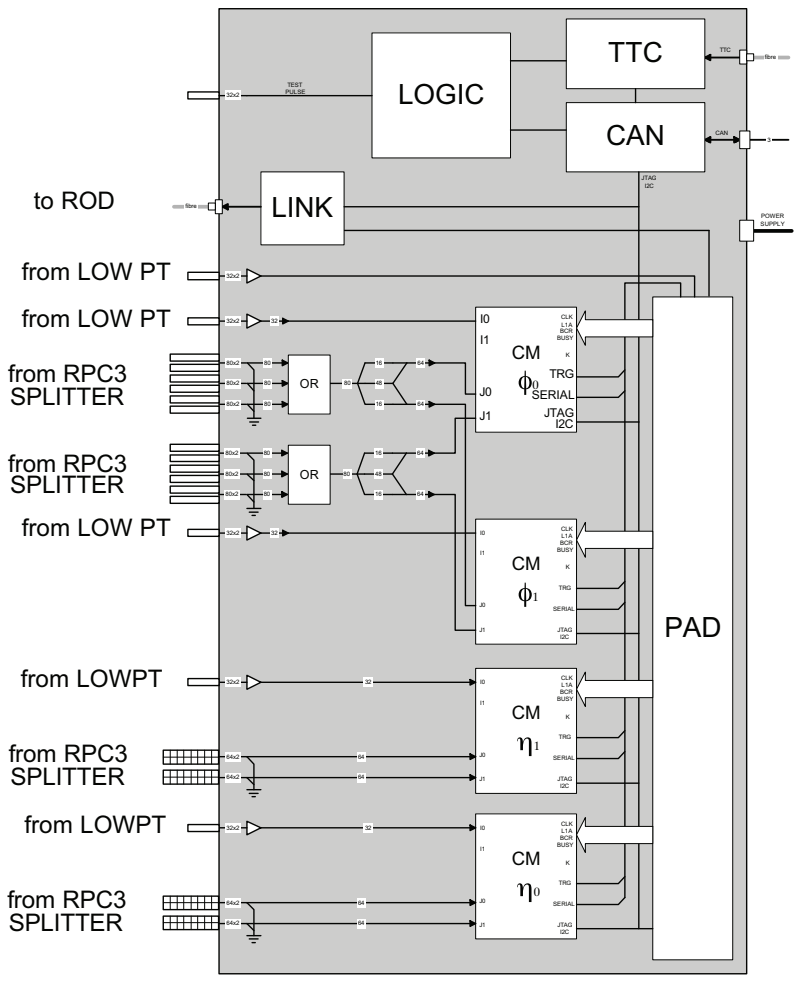

Figure 13. PAD high layout. The PAD logic implemented in an FPGA processes readout and trigger data and sends the results to an output link

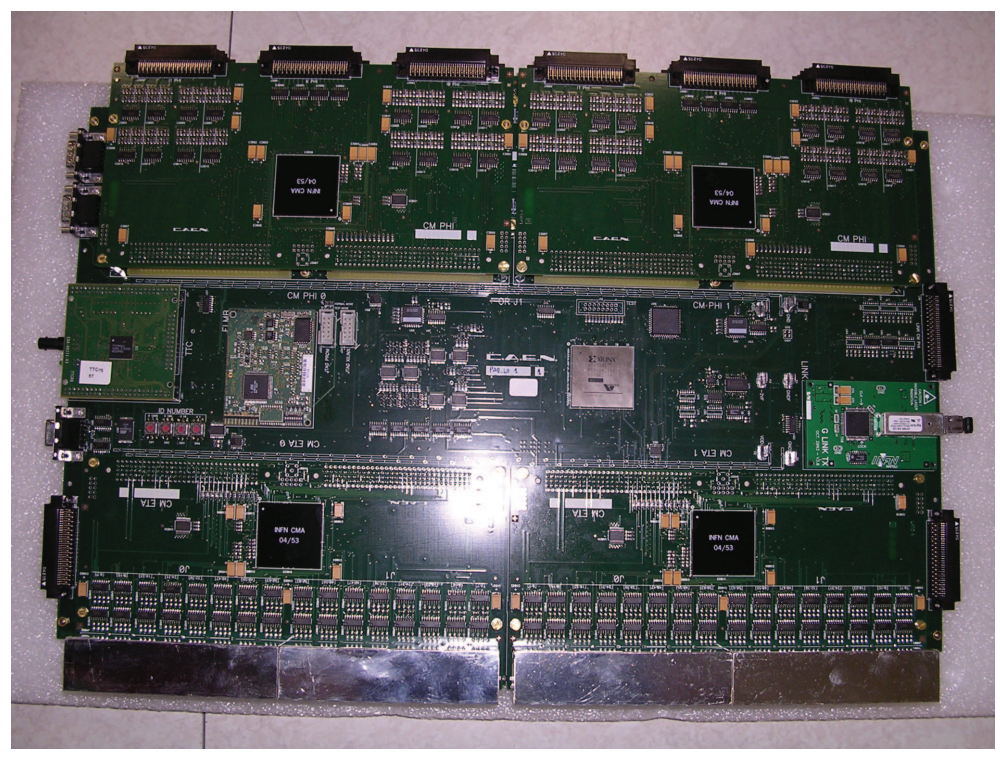

Figure 14. picture of a PAD out of its encasing. On the lower part the two $\eta \mathrm{CMs}$ addon boards can be seen. In the central part, starting from left: TTCRQ, ELMB,PAD FPGA, Optical link mezzanines can be seen. In the lower part the two $\phi$ CMs add-on boards can be seen 


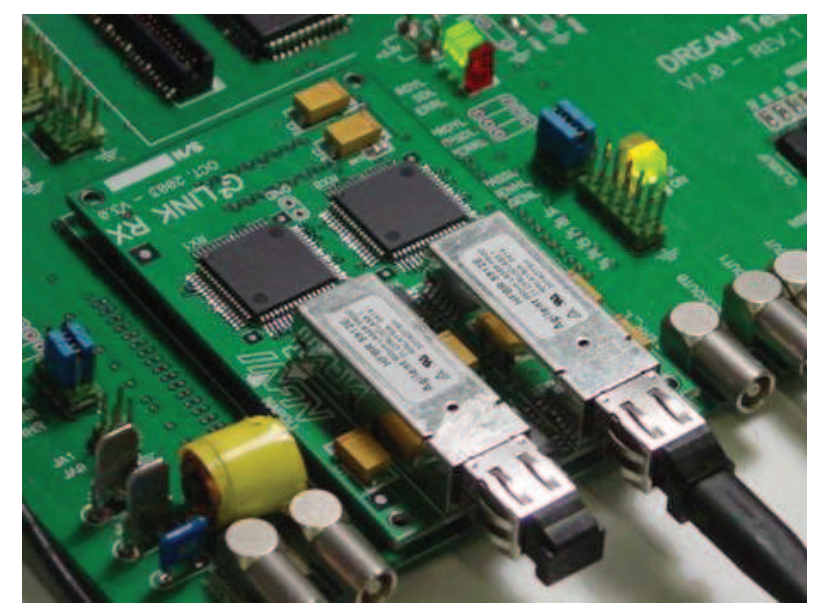

Figure 15. Photograph of a receiver Glink board (RX)

total, the Muon Trigger needs 500 Tx nodes and 250 Rx nodes. Despite the availability on the market of new-generation serializer/deserializer chipsets, we have selected for this purpose the G-Link HDMP-1032A/4A [17] for their ability to guarantee a fixed latency.

In the ATLAS environment, all the electronics (including the Tx and Rx optical nodes) share a replica of the Large Hadron Collider (LHC) clock via an ad-hoc distribution tree. The frequency-agile architecture of the G- Link chipset is not bound to specific data rates and it allowed us to use this clock $(40.078 \mathrm{MHz}$ ) for the transmission. The bit-rate on the fibre is 20 times higher ( $800 \mathrm{Mbit} / \mathrm{s})$, with a time slot for each bit (Unit Interval or UI) of $1.25 \mathrm{~ns}$. The PASS circuitry makes it possible to implement a true fully-synchronous link: the Tx strobes the input bus and the Rx drives the output bus by using the same LHC clock. The latency is constant and not influenced by environmental parameters and fabrication process variations. It does not change after a reset, a loss of lock or even a power cycle. This feature is critical for the correct operation of the L1 Muon Trigger, which works in a pipeline fashion synchronous to the LHC clock. We also benefit from the G-Link capability to distinguish between data and control words (differently codified in the serial stream). This allows us sending across the link two different logical streams, one assigned to the RPC detector read-out (data words, 16-bit payload), the other to the L1 Muon Trigger (control words, 14bit payload). At the present time, no other commercial architecture offers comparable features. For these reasons, the G-Link has been widely used also in other ATLAS detectors, both as HDMP-1022/4 and HDMP-1032A/4A.

\subsection{The off-detector Trigger and Readout System}

On-detector electronics executes the trigger algorithm and send the relevant trigger information, via optical links, to the off-detector Central Trigger Processor, that can validate or reject the event with a fixed latency of $2.5 \mu \mathrm{s}$. During the decision time of 


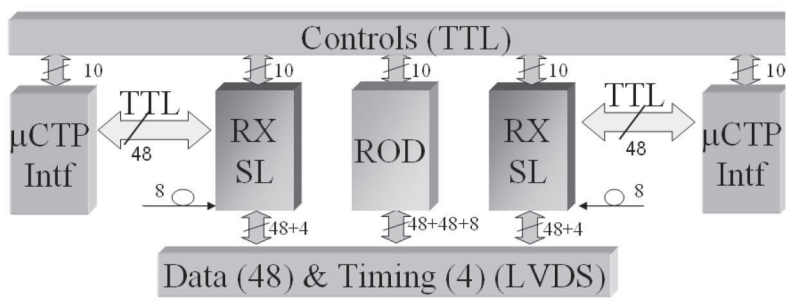

Figure 16. Block diagram of all parts on the off-detector system, including the MUCTPI Interface boards, the RX/SL board, the ROD board and the RODbus backplane connections, carrying Controls, Data and Timing signals

the Trigger processor, data produced by the RPC detectors are stored in FIFO memories on the on-detector electronics. If an event is accepted by the first level trigger, a L1A pulse is generated and transmitted across the TTC system together with the pertinent EVID and BCID; in this case, data stored in the FIFO buffers are transferred to the off-detector electronics across the same optical link used for the transmission of trigger information. Trigger and read-out data of each sector of the spectrometer are managed by a Read Out Driver (ROD) crate. RX-SL boards have the task to receive and elaborate trigger and read-out data from the on-detector electronics. The RX/SL boards preprocess the trigger information and send them to the Central Trigger Processor through the Barrel Muon Central Trigger Processor Interface board (MUCTPII). The RX/SL boards arrange readout data in an event frame (RX Frame) and transmit them to the adjacent Read Out Driver across a custom backplane RODbus via a high speed serial link.

The main task of the ROD [36] is to perform a further framing, before transmitting data across the optical link S- Link to the next acquisition levels, i.e. to the Read Out Buffers (ROB). The ROD also manages the timing signals of the trigger and data acquisition system. For this purpose, the ROD hosts a TTCrq receiver module from which it receives timing and control signals to be forwarded to the RX/SL boards on the RODbus. On the RODbus, data and timing signals are transmitted in LVDS standard to achieve high rate, low skew and jitter; the serial links between each RX/SL and ROD have an aggregate bandwidth of 2 Gbit/s (48bit@40MHz). Control signals run at lower rate and are transmitted using the TTL standard. The RODbus also hosts a 48-bit TTL bus that allows the RX/SL boards to transmit trigger data to the CTPI boards. The ROD boards developed for the other ATLAS subdetectors implement different logic and functionalities, in order to fulfil the specific detector requirements. However, they share the same logical output format and optical fibre. In this way, the ROB design is unique for the entire apparatus, making it possible to use the same architecture for the higher level DAQ systems. 


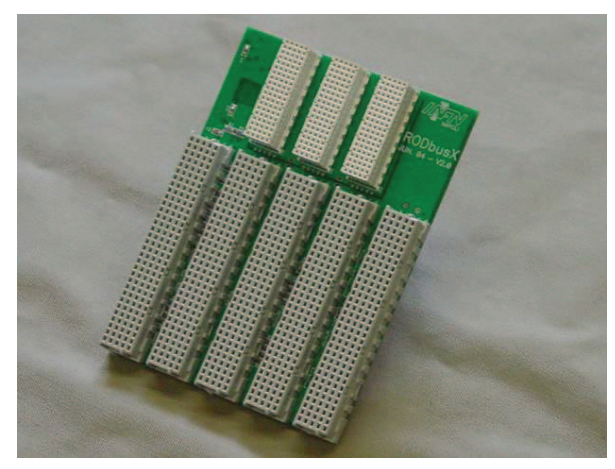

Figure 17. picture of a RODbus, before insertion in the VME64x backplane. Two of these buses are mounted on a 21-slot VME crate

\subsection{The Sector Logic (SL)}

The SL board has a form factor of the VME $64 \mathrm{X} 6 \mathrm{U}$ standard and is equipped with two VIRTEX II Xilinx FPGAs, labelled VME FPGA and SL FPGA. The board also hosts one 32-bit 8k word deep FIFO, up to four Glink two-channel receivers (RX mezzanine), and one Serializer (TX SerDes) that sends data via RODbus backplane to the adjacent ROD [27]. The main task of the VME FPGA is to initialize and control the four RX mezzanines and to interface the whole board to the VME bus. It is possible to access its own configuration registers and the SL FPGA internal registers. The VME FPGA is also capable of driving a JTAG chain to download new versions of the SL firmware. This procedure greatly simplifies the number of operations when new firmware has to be downloaded into all SL of the experiment.

The board clock can be obtained either locally or from the TTCRq mounted on a neighbouring ROD, received via the RODbus. Other timing signals like L1A, BCR and ECR are received with the same mechanism. The event building part of the SL FPGA internal clock domains are normally working at double of the system frequency. The SL FPGA performs event building on data received by all PADs belonging to a trigger sector. Normally 6 or 7 streams of 16 -bit words are received by the RX mezzanines and processed by an event building state-machine which has been built with some tolerance to transmission errors, i.e. if words are received from a data source which are not compatible with the data format, they are discarded until a proper data frame arrives. This process continues until a maximum number of retries is reached. In this case the event fragment is built with error flags. An important debugging tool to monitor the trigger system is the presence, inside the event fragment, as built by the SL, of all input and output trigger data belonging to the trigger path of the event. Clock domains belonging to the input data, the event building and the data transmission parts are decoupled inside the SL FPGA by extensive use of double-clock FIFO's. VME and SL FPGA communicate with a simple 32-bit data 4-bit control word parallel synchronous protocol, where the VME FPGA is always considered as master for the transmission. The on-board FIFO is also connected to the VME FPGA and its main purpose is monitoring processed data. 


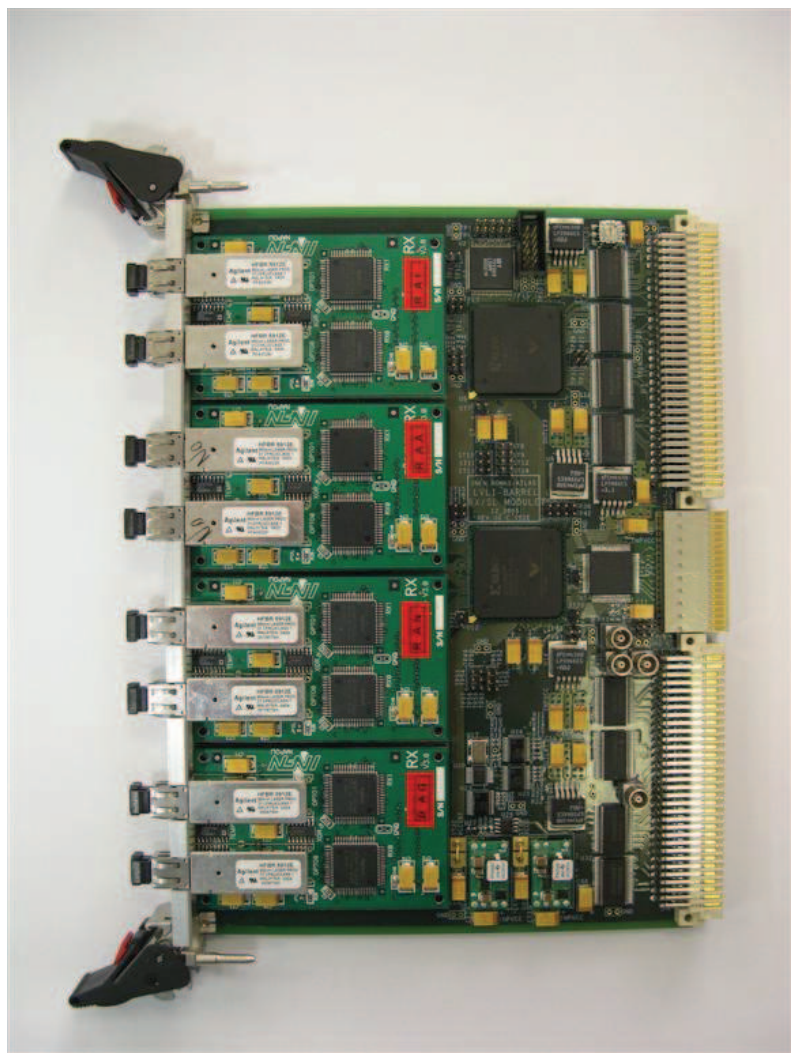

Figure 18. Photograph of a Sector Logic (SL) board. The four RX optical link mezzanines are mounted on the left, while the VME FPGA is on the upper right side. In a central position, the SL data processing element can be seen, while the serializer shipping data to the ROD is very close to the central backplane connector

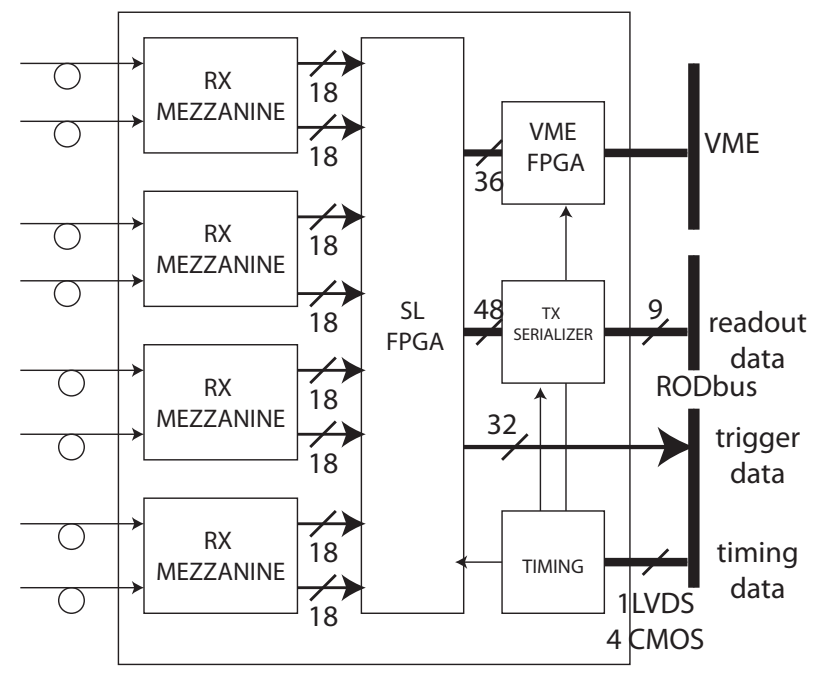

Figure 19. Block diagram of a Sector Logic (SL) board. Trigger and readout data are received by the RX mezzanizes and sent to the SL FPGA. The VME FPGA is responsible for configure and control via the VME bus. 


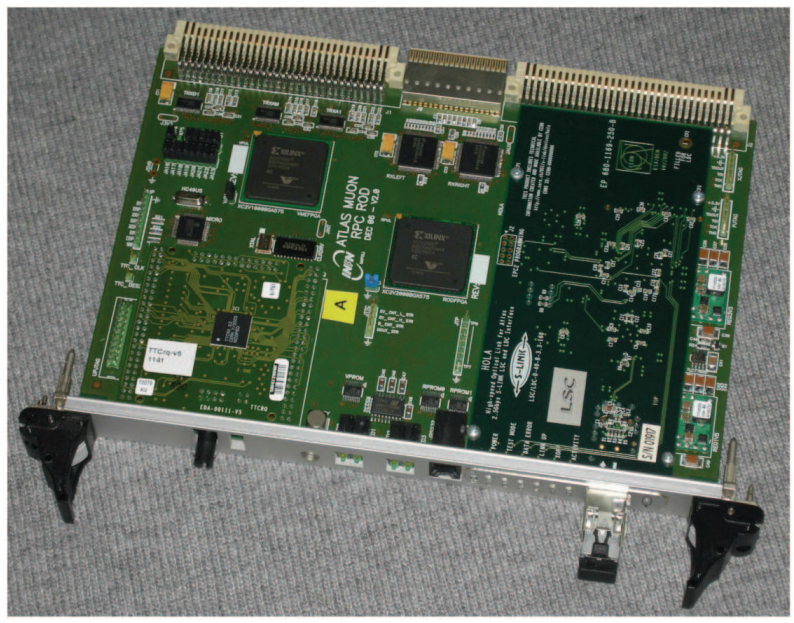

Figure 20. Photograph of a ROD

Regarding the trigger part, the optical link receiver assures a fixed latency delivery of trigger information from the on-detector electronics. A programmable delay of up to $3 \mathrm{BCs}$ is built at each input to synchronize if necessary the trigger towers belonging to the same beam crossing. Within a latency of $7 \mathrm{BCs}$, data from up to 8 sources are processed and the two highest $\mathrm{Pt}$ candidates are available, if present, on the 32-bit output word. All relevant information, i.e. details on RoI, Pt value overlap flags are sent to the MUCTPI interface.

\subsection{The Readout Driver}

The ROD board (Fig. 20) has the form factor of the VME 64X $6 \mathrm{U}$ and is equipped with two VIRTEX II XILINX FPGAs, labelled as VME FPGA and ROD FPGA. The board also hosts an ARM7 microcontroller, the TTCrq receiver, the S-Link transmitter and the two deserializers (RX SerDes) that receive data via RODbus backplane from the RX/SL boards. The main task of the VME FPGA is to interface the whole board

with the VMEbus; the VME FPGA allows a user to access the ROD FPGA memory locations and configuration registers and to read the microcontrollers data. The VME FPGAs clock is obtained from an on-board $40 \mathrm{MHz}$ oscillator multiplied by 2 by the internal Digital Clock Manager. The ROD FPGA performs the event building algorithm on data transmitted by the RX/SL boards. The ROD FPGA also hosts registers for the configuration and control of the event builder state machine. In the same fashion as the VME FPGA, the ROD FPGA clock is obtained multiplying by 2 the $40 \mathrm{MHz}$ board clock. The ROD FPGA receives 48-bit words and the recovered $40 \mathrm{MHz}$ clock from each RX SerDes. It is also interfaced with the TTCrq module - from which it receives the TTC timing signals and the $40 \mathrm{MHz}$ LHCs clock - and to the S-Link transmitter, that is fed by a $40 \mathrm{MHz}$ clock derived by the $80 \mathrm{MHz}$ internal clock.

Fig. 21 shows a simplified block diagram of the ROD board. The ROD FPGA communicates with the VME FPGA via a serial synchronous custom protocol, carried 


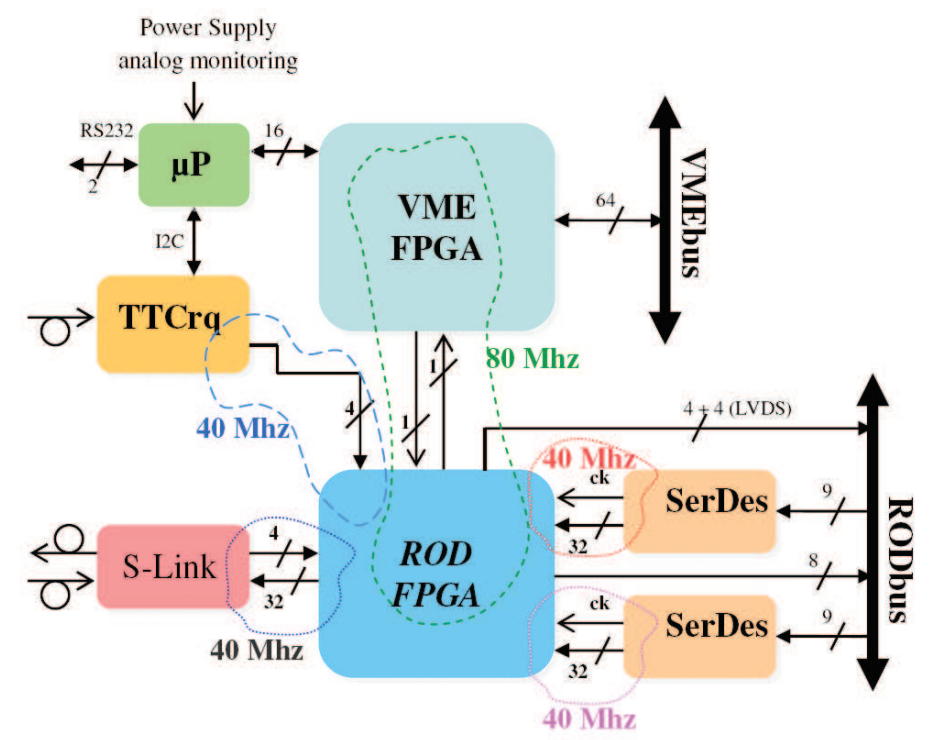

Figure 21. Block diagram of a ROD

out by two point-to-point unidirectional lines with a data rate of $80 \mathrm{Mbit} / \mathrm{s}$. The main advantages of a serial link are a simpler PCB layout, the use of a small number of FPGA pins and limitations of ground bounce effects. The VME FPGA is the Master of the serial link, managing both the write (for data and for address) and read operations. As a consequence, the ROD FPGA can transmit data only if the VME FPGA has previously requested them. The serial protocol allows the user to set once an 8-bit target address and then to perform an arbitrary number of 32-bit read or write accesses to the selected location in the ROD FPGA. The main task of the ARM7 microcontroller is to program the TTCrq receiver, via $\mathrm{I} 2 \mathrm{C}$ protocol. This makes it possible to access all the TTCrq registers, both for configuration and monitoring purposes. The microcontroller also allows reading, via the internal ADC, the three power supplies on the ROD board $(5 \mathrm{~V}$, $3.3 \mathrm{~V}, 1.5 \mathrm{~V})$. The power supply and temperature on the RODbus are acquired from a remote ADC installed on the backplane via an $\mathrm{I} 2 \mathrm{C}$ bus. The microcontrollers output data can be read via a RS232 port or can be redirected on the VMEbus, through a 16-bit parallel bus handled by the VME FPGA. Besides the internal $80 \mathrm{MHz}$ FPGA clocks, the $40 \mathrm{MHz}$ LHC clock, the two $40 \mathrm{MHz}$ SerDes clocks, the $40 \mathrm{MHz}$ S-Link clock run all over the board. Even if these clock signals have the same frequency, they have an unpredictable phase relationship and should be handled as domains asynchronous to each other. All these clocks are present in the ROD FPGA, which is the most complex and critical section of the board. In order to decouple the clock domains and to guarantee their coexistence on the ROD FPGA, FIFO memories have been extensively used. 


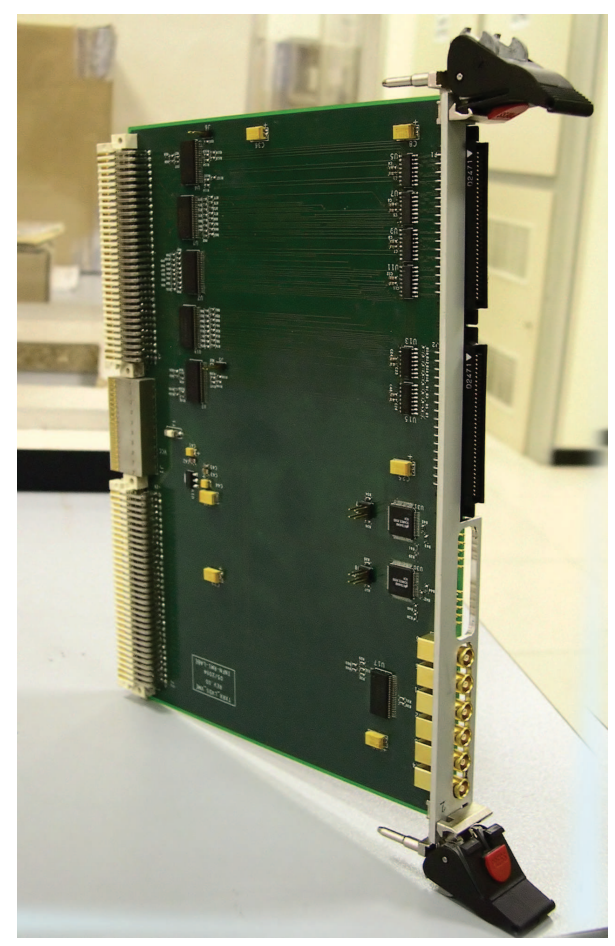

Figure 22. Photograph of a Barrel MUCTPI Interface board

\subsection{Barrel MUCTPI Interface}

The main function of the MUCTPI Interface is to interface the SL trigger output to the MUCTPI boards sitting in a crate about $10 \mathrm{~m}$ apart. This is accomplished by converting the CMOS SL output into a 32-bit LVDS bus. The interface also contains a set of LVDS receivers. These receivers connected to the SL via RODbus could be used in a future trigger upgrade and used as inputs from Tilecal hadronic calorimeter.

\subsection{System interconnections}

The decision taken on the choice of the system interconnections has been to use copper where distances were less than $10 \mathrm{~m}$ and optical connections elsewhere, in particular between the cavern and the counting room.

After having built a database holding lengths and cabling maps for all so-called $\eta$ and $\phi$ cables, connecting Splitter to PAD modules, a dedicated program has been running on laptops used by the cabling operator in the cavern. This program accessed the cabling database to produce a detailed cabling procedure. This procedure guided the operator through cabling showing graphically the cabling sequence, i.e. source and destination connectors for each of the many thousands of cable of different type and length that were coped for by using this program.

Other system connections include the so-called low-to-high cables, arranged in bundles of 6 32-twisted-pairs and connecting the low to the high $p_{T}$ PADs. These are custom built twisted pair shielded cables. 


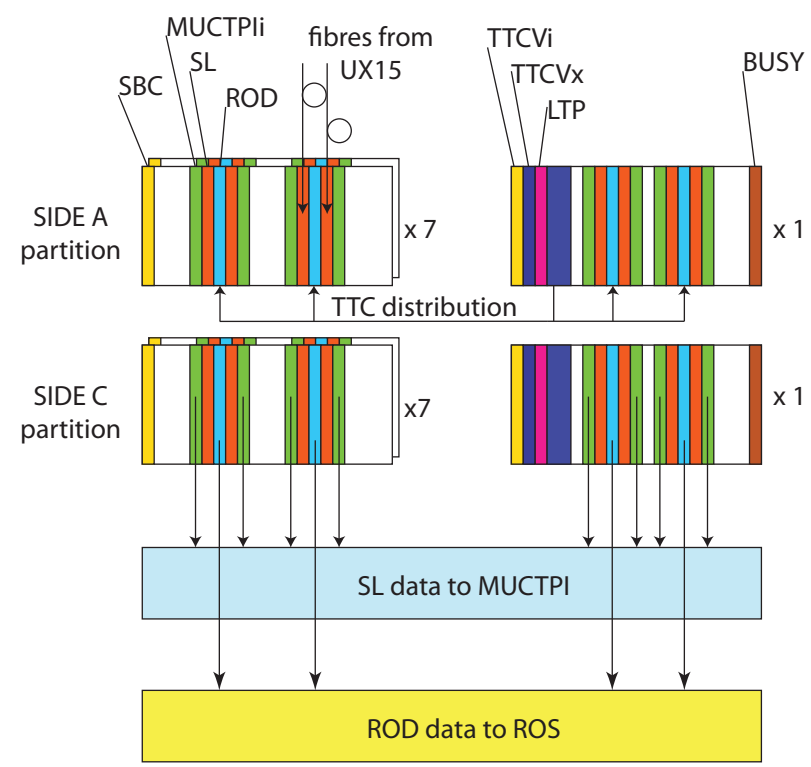

Figure 23. Arrangements of all VME crates in two TTC partitions. For clarity connections are shown only on a few modules per type

Another important connection type is the control network, where all PADs belonging to a station are connected in daisy chain via a CANbus connection. A total of 64 of these custom built cables make up the network, each connecting up to 14 nodes arranged in a daisy chain. They have been laid and connected to 8 dedicated control PCs, each hosting a quad KVASER CANbus controller card.

The final system also contains a Timing and Trigger distribution network, organized in two independent partitions. The clock and other timing signals are propagated from the TTC modules to the PADs, with optical fanouts of 1-to-16 in USA15 and 1-to-8 in the cavern, and to the RODs, with an optical fanout of 1-to-16, and finally from the RODs to SL modules using the RODbus.

\subsection{The power system}

The power system design has been done with the following requirements in mind, namely the need for radiation tolerance and the need to operate in presence of magnetic field.

The necessity of putting the system close to the detector, thus reducing cable lenghts and cost, has put these constraints which have ruled out the choice on many off-the-shelf systems.

The final choice has gone on the CAEN EASY system. This industrial system has been designed to survive to the ATLAS cavern environment, in presence of radiation and magnetic fields of about 300 Gauss.

It consists of a set of tri-phase to $48 \mathrm{~V}$ DC power generators and custom crates containing all types of modules needed:

- 2-8V, 25A modules 
- 2-8V 9A modules

- ADC modules

A control chain connects all crates together to a mainframe controller sitting in the counting room. Since the trigger and detector parts are tightly integrated and under control of the RPC detector DCS system, no further details will be given here. To give an idea of the system size, around 140 modules power the on-detector electronics of the Level-1 system.

The total dissipated power of the trigger system is about $8 \mathrm{~kW}$, with about $20 \%$ of this value dissipated on cables.

\subsection{Environmental issues}

2.14.1. Radiation tolerance All on-detector components used in the system have been evaluated for the irradiation doses expected in the experiment [30], [28], [29]. The Barrel worst case values are total ionizing dose of 3.04 Gy in 10 years, and a Single Event Effects

(SEE) worst case: $5.65 \times 10^{9} \mathrm{~h} / \mathrm{cm}^{2}$ in 10 years. This requirement has lead to a long validation study of all devices used in the system, with dedicated irradiation campaigns. Results can be found on measurements done at Louvain (Belgium), PSI (Switzerland), where components where irradiated with $60 \mathrm{MeV}$ protons, in Italy where components where irradiated with a $C o^{60}$ source. All components chosen for the final system have shown resistance up to the expected doses.

\section{Construction}

The construction of the building blocks of the level-1 Muon Barrel system has gone through many steps, from the evaluation of prototype to Preliminary, Final, Production Readiness reviews. Without going into detail for the full system, the on-detector electronics has gone through the following tests after production:

- Industrial test: this test has been done in Bologna using an industrial JTAG system connecting each of the produced 832 PAD boards to dedicated test boards. The daisy chain thus built had almost full coverage of the board IOs. The test program was able to show where the fault was and its type. Voltages and temperatures were readout also during this test, together with the phase measurements of the delay chips. This test showed that about $17 \%$ of the production needed to be reworked (bad solderings, misplaced or missing components were found).

- Functional test: this test has been done at CERN to modules passing the previous test. In this case a full functional test included the readout of the data after sending test pulses to all inputs. Programming of the FPGA and full initialization were also done.

- Integration test: in this case the PAD and Splitter boxes have been integrated to the RPC and Monitored Drift Tube chambers. A cosmic test stand has been used to 
check the behaviour of the system. Chambers were operated with final gas mixture and Voltage, and the readout operated in final working mode [18]. These tests were useful also to find cabling errors, discover dead channels. More that $350 \mathrm{k}$ channels were tested this way, finding only $0.46 \%$ of dead channels [32],[37].

- Chamber tests have been carried out in the ATLAS surface installation area to each chamber before lowering it in the pit. A testpulse was used in this case to check if the full readout and trigger chain was fine after transport.

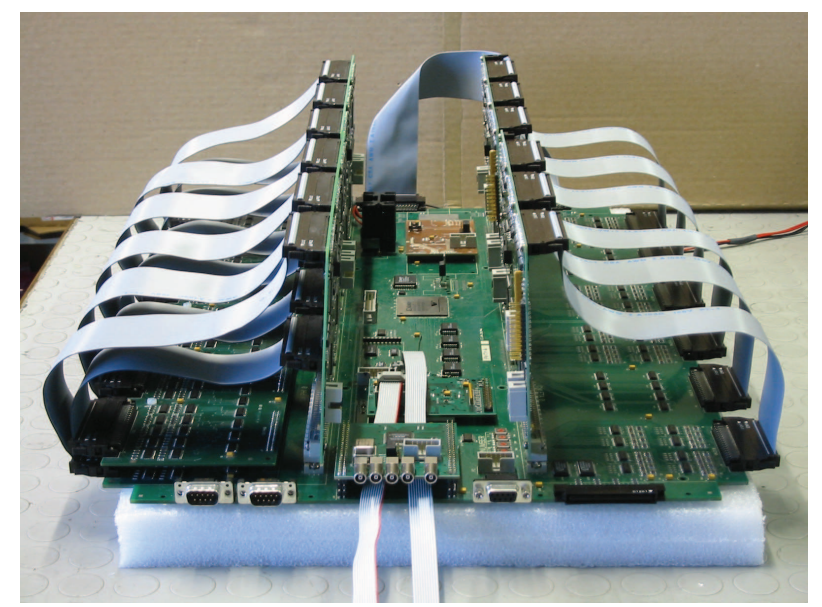

Figure 24. Photograph of the PAD industrial test system.

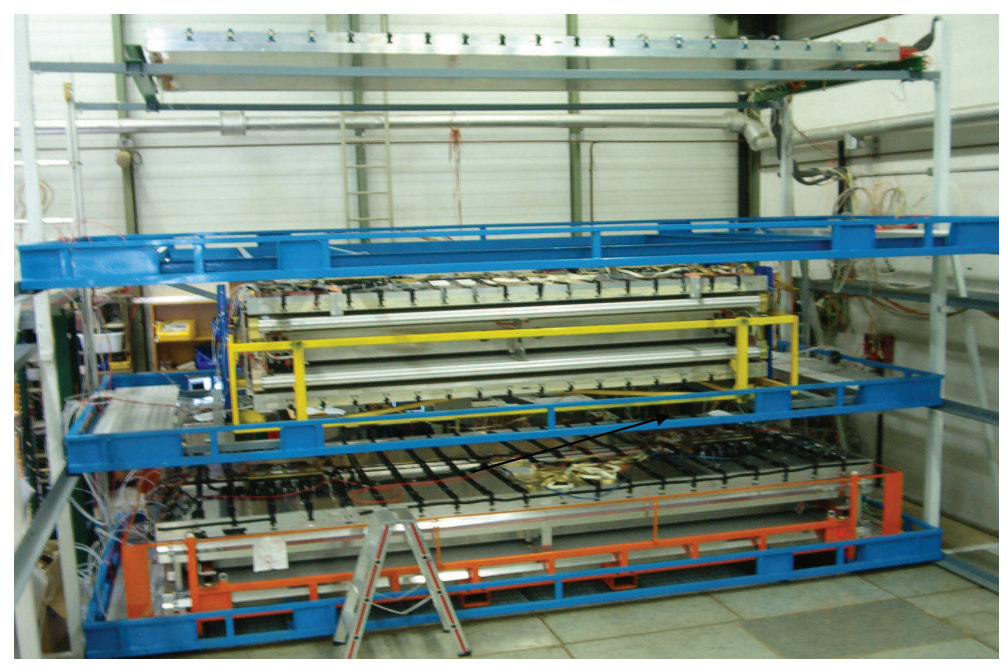

Figure 25. Photograph of the cosmic stand.

\section{The initialization and control system}

The Level-1 Control software runs on two platforms communicating via the CANbus, the first platform is a linux-based PC running dedicated software based on the ATLAS 


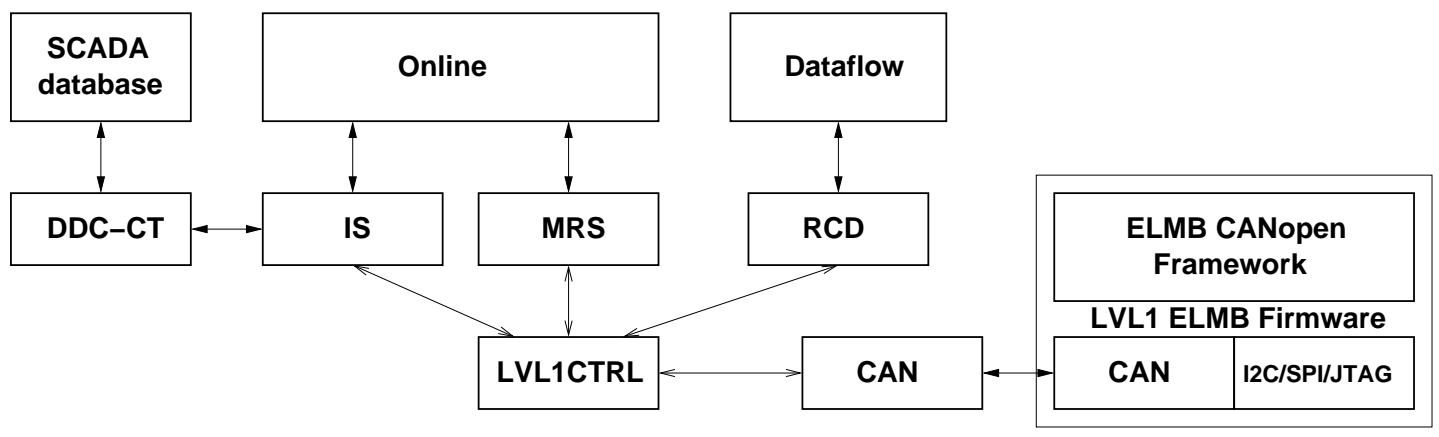

Figure 26. Block diagram of the interaction between, DAQ, DCS, the LVL1 Control Application and the PAD processor.

Online and Dataflow framework while the second is microcontroller-based, running custom software using the ELMB CANOpen firmware framework [13]. Both platforms make use of a common set of CANOpen commands (Object Dictionary), which has been customized to work in two different modes.

- handle single bus transactions;

- issue specific commands like Initialize or Check PLL locks.

They exchange Service Data Object (SDO) packets. The number of CANopen packets which are sent to complete a single bus transaction is shown in Tab2, while specific commands require only a single CANopen packet.

Table 2. Number of SDO packets per local command

\begin{tabular}{|l|c|}
\hline Bus transactions & No SDO packets \\
\hline I2C register RW & $3-4+($ nbytes $\div 4)$ \\
SPI memory RW & $3+($ nbytes $\div 4)$ \\
JTAG block RW & $4+($ nbytes $\div 4)$ \\
\hline
\end{tabular}

\subsection{Integration with $D A Q$}

A control and initialization Module has been implemented in the ATLAS DAQ system [35], this application is able to react to commands coming from the Run Control (LOAD, CONFIG, START, UNLOAD) and issue the required actions to the on-detector electronics. Errors during configuration are reported via the Message Reporting System (MRS) to the DAQ console. After start of run, the Level-1 Control application communicates to DCS, via DAQ-DCS Communication Software (DDC), periodic measurements and status of the system. No direct connection is foreseen between Level-1 Control PCs and the ATLAS DCS system database (SCADA). Fig.26 shows the connections of the Module to the DAQ and DCS system.

The online software needs to provide to each control PC the static maps of nodes connected to it. 


\subsection{System Configuration}

Configuration of the system can be done in two ways, either using a list of bus transaction commands, or using generic commands implemented in the ELMB firmware. The first mode is used mainly for debug purposes while the second is used for fast initialization and control in the experiment. A standalone application is capable of handling all possible commands defined in the Object Dictionary, and to combine them for specific purposes. A logging procedure is able to write on file the list of atomic commands issued to the CAN node for initialization. These log files are used to build ordered sets of command lists. Each set can be optionally compiled in order to produce a binary file, following the configuration command scheme described below, which is stored locally in the PAD Processor flash memory, to be executed during initialization. The local flash memory size, of $8 \mathrm{Mbit}$, is large enough to contain more than one initialization type. Thus during normal data taking, a single CAN bus transaction, initialize the Processor for different Cosmics or Beam run types, setting timing, thresholds, coincidence roads. Each configuration is stored in different locations of the local memory, and can be started with a single command of the Object Dictionary.

Since the partitioning of the system permits to initialize all CAN buses in parallel, we expect a total fast initialization time of a few seconds for the full barrel system.

The structure of the configuration is shown in Fig.27. A configuration header is followed by a number of variable-length fields. Each field is a command line transformed by the microcontroller into an $\mathrm{I} 2 \mathrm{C}$ register access. The result of each individual access is stored and at the end of execution is available to the control PC.

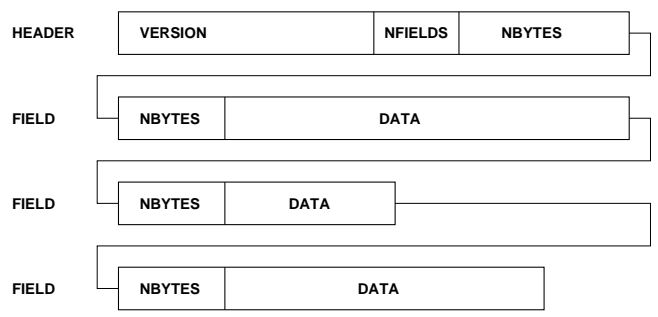

Figure 27. Structure of configuration stored in local memory.

\subsection{Configuration database}

Each configuration of the Level-1 trigger system is stored into a hierarchy of flat ASCII files. The Configuration database contains these files, their binary version and the list of configurations currently loaded locally on the system. If the configuration required by DAQ during CONFIG is locally stored a fast initialization will start, otherwise the Level-1 Controller will retrieve the required set of files, tagged by run type and time interval of validity, and a download will be issued to all nodes. The same procedure is required each time a change in the configuration is needed. 
The current configuration status of the system must be known at any time. If the Control PC configuration files need to be changed, they have to be retrieved from the database. If new configuration need to be stored on the on-detector processors, the ASCII configuration files need to be processed, then a download will be issued to all nodes.

This operation is time consuming and should be done some time before the configuration is required, not to interfere with the data acquisition.

The structure of the configuration files reflects the system architecture, organized in sectors, towers and PAD processor, and at the lowest level each PAD processor is assigned a concatenation of device-specific configuration files. This structure is also functional to calibration programs, who need to know the current configuration, process calibration or normal data, to produce new configuration files.

\subsection{System Monitoring}

A second requirement to the Control system is the monitoring of the system during data taking. All PAD processors make available during data taking the following information:

- main power supply Voltages, all regulated Voltage levels;

- temperatures of all critical devices and inside the PAD and Splitter boxes;

- trigger rates on bending and non-bending projections;

- status of filling of memories of the readout chain;

- alarm flags of overcurrents due to Single Event Effects latchup;

- lock status of TTC receiver, ASIC, FPGA, delay chips and transmitter.

All this information has to be made available together with the RPC chamber condition data. The tool used to propagate the information from the DAQ to DCS has been developped within the ATLAS Online software Group, and it is called DDC (DAQ DCS Communication). It uses the infrastructure of the Online Information service.

\section{Latency}

A key parameter of the level-1 system is the trigger latency, time delay imposed to the front-end electronics before sending the read-out data. This value has to be fixed and given by processing time plus propagation along the cabling system. Maximum acceptable value in ATLAS is $2.5 \mu \mathrm{s}$, equal to 100 bunch crossings. In table 3 the various contributions to the latency are shown. It has to be kept in mind that the difference in length between the fibre bundles carrying trigger signal from cavern to counting room is the source of uncertainty in the table shown. During normal operations these differences are absorbed by proper settings of the MUCTPI input pipelines. 
Table 3. Contributions to RPC trigger latency

\begin{tabular}{|l|c|}
\hline$\Delta t$ & Delay contribution \\
\hline TOF to middle station & $50 \mathrm{~ns}(15 \mathrm{~m})$ \\
\hline optical fibres transmission & $252 \div 401 \mathrm{~ns}(51 \div 81 \mathrm{~m})$ \\
\hline LVL1 Muon Barrel processing time & $600 \mathrm{~ns}$ \\
\hline SL-MUCTPI connection & $50 \mathrm{~ns}(10 \mathrm{~m})$ \\
\hline MUCTPI-CTP output & $290 \mathrm{~ns}$ \\
\hline L1A Latency & $252 \div 421 \mathrm{~ns}(51 \div 85 \mathrm{~m})$ \\
\hline MUCTPI input & $952 \div 1101 \mathrm{~ns}$ \\
\hline CTP output & $1242 \div 1391 \mathrm{~ns}$ \\
\hline
\end{tabular}

\section{Conclusions}

At the time of writing the entire Barrel Muon trigger system has been installed, both on and off-detector. The system has partecipated to a long series of integration runs with the rest of the experiment which have finally brought to the common runs at the LHC startup of 2008. The trigger is being commissioned with cosmic-ray, where calibration runs are being performed to prepare the system for the LHC 2009 running.

\section{Acknowledgments}

This system has taken many years of intense work of development, first in the design phase, then during construction and installation [26], [24], [21], [19] [31], [20]. We wish to thank all engineers, technicians and students which have contributed during these various phases. The advice of many people from the ATLAS community has been very helpful, we wish to thank in particular Nick Ellis, who has given us support and advice over many years. We wish to thank Philippe Farthouat for his advice during all phases of development of the electronics. Close collaboration with collegues of the RPC community has been very stimulating for the design of the system, it has now brought to the actual merging of detector and trigger communities into one. We wish to thank CAEN and in particular Andrea Romboli and Carlo Tintori for the PAD processor engineering and the development of the printed circuit boards. Authors wishing to acknowledge all INFN engineers and technicians for the enormous amount of work done, in particular Antonio Anastasio, Giacomo Chiodi, Felice Cidronelli, Silvano Di Marco, Ettore Gennari, Riccardo Lunadei, Pasquale Parascandalo, Antonio Rossi, Daniele Ruggieri and Alessandro Ruggieri. The Level-1 Muon Barrel Trigger has been supported by the Istituto Nazionale di Fisica Nucleare.

\section{References}

[1] "the ATLAS Fist-Level Trigger Technical Design Report", 
CERN/LHCC/98-14

ATLAS collaboration.

[2] "Muon Spectrometer Technical Design Report" CERN/LHCC/97-22

ATLAS collaboration.

[3] ATLAS Level-1 muon trigger Group , "Improvements to the Level-1 muon trigger giving increased robustness against backgrounds"

ATL-COM-DAQ-99-011.

[4] "The ATLAS First-Level Muon Trigger in the Barrel", ATL-MUON-INT-2007-002

F.Ceradini et al., (Reading, MA: Addison-Wesley)

[5] "ATLAS Level-1 muon barrel trigger robustness study at X5 test facility"

A. Di Mattia et al.

Nucl. Instrum. Meth. A 518, 529 (2004)

Prepared for 9th Pisa Meeting on Advanced Detectors: Frontier Detectors for Frontier Physics, La Biodola, Isola d'Elba, Italy, 25-31 May 2003

[6] "The ATLAS RPC level-1 muon trigger: Design and simulation"

Published on Nuclear Instruments \& Methods, A 533 (2004) 188

Proceedings of Workshop on Resistive Plate Chambers and Related Detectors (RPC2003),

Clermont-Ferrand, France, 20-22 Oct 2003

[7] "PYTHIA manual", http://home.thep.lu.se/ torbjorn/pythia/lutp0613man2.pdf

[8] "DPMJET manual", http://arxiv.org/abs/hep-ph/9911232v1

[9] "The RPC Level-1 Muon Trigger of the ATLAS Experiment at the LHC" Published as ATLAS Note ATL-CONF-2006-004

Proceedings of the 14th IEEE - NPSS Real Time Conference 2005 (RT2005), Stockholm, 4-10 Jun 2005

[10] "The ATLAS Level-1 Barrel Muon Trigger Performances"

Proceedings of 2005 Nuclear Science Symposium and Medical Imaging Conference (2005 NSS/MIC), Puerto Rico, October 23 - 29, 2005 (R.Vari)

[11] "The Muon Spectrometer Barrel Level-1 Trigger of the ATLAS Experiment at LHC "

Published on IEEE Trans. Nucl. Sci. 53 (2006) 2446.

Proceedings of 2005 Nuclear Science Symposium and Medical Imaging Conference (2005 NSS/MIC), Puerto Rico, October 23 - 29, 2005

[12] "Performances of the Coincidence Matrix ASIC of the ATLAS Barrel Level-1 Muon Trigger"

Published as ATLAS Note ATL-CONF-2006-017

Proceedings of the 11th Workshop on Electronics for LHC Experiments, Heidelberg, Germany, 12-16 Sept 2005 (R.Vari)

[13] "http://atlas.web.cern.ch/Atlas/GROUPS/DAQTRIG/DCS/ELMB/ELMBhome.html."

[14] "The RPC First Level Muon Trigger In The Barrel Of The Atlas Experiment"

Published on Nuclear Physics B, Proc. Suppl. 158 (2006), p 11-15

Proceedings of 8th Workshop on Resistive Plate Chambers and Related Detectors, Seoul, Korea, 10-12 Oct 2005

[15] "Performances of the ATLAS Level-1 Muon Trigger processor in the Barrel" Published as ATLAS Note ATL-COM-DAQ-2006-033

Proceedings of the 9th ICATPP Conference, Villa Olmo - Como, Italy, 17-21 October 2005 (Fr.Pastore)

[16] "The first-level trigger of ATLAS" 
Published as ATLAS Note ATL-DAQ-PUB-2006-001

Proceedings of European Physical Society (EPS) International Europhysics Conference on High Energy Physics, Lisbon, Portugal, 21-27 Jul 2005

[17] "Do's and Don'ts with the Agilent's G-Link Chipset"

A. Aloisio et al.

Proceedings of the 2005 IEEE Nuclear Science Symposium, Portland.

[18] "The trigger chambers of the ATLAS muon spectrometer: Production and tests" Published on Nuclear Instruments \& Methods, A 535 (2004) 265

Proceedings of 10th Vienna Conference on Instrumentation, Vienna, 16-21 Feb 2004

[19] "The RPC LVL1 trigger system of the muon spectrometer of the ATLAS experiment at LHC"

Published on IEEE Trans. Nucl. Sci. 51 (2004) 1581

Proceedings of the 13th IEEE - NPSS Real Time Conference (RT 2003), Montreal, Canada, 18-23 May 2003

[20] "Test Beam results and integration of the ATLAS Level-1 Muon Barrel Trigger" Published as ATLAS Note ATL-DAQ-CONF-2005-012

Proceedings of the 2004 Nuclear Science Symposium and Medical Imaging Conference, Rome 2004 (Fr.Pastore)

[21] "Test beam results from the ATLAS LVL1 Muon Barrel Trigger and RPC readout slice"

Proceedings of the 2003 Nuclear Science Symposium and Medical Imaging Conference, Portland, Oregon (U.S.A.), October 19-25, 2003 (S.Veneziano)

[22] "Slice Test Results of the ATLAS Barrel Muon Level-1 Trigger"

Published as ATLAS Note ATL-DAQ-2003-054

Proceedings of the 9th Workshop on Electronics for LHC Experiments, Amsterdam, Holland, 29 Sep - 3 Oct 2003 (R.Vari)

[23] "The Coincidence Matrix ASIC of the Level-1 Muon Barrel Trigger of the ATLAS Experiment"

Presented to the 2002 Nuclear Science Symposium and Medical Imaging Conference, Norfolk, Virginia, 10-16 November 2002 (R.Vari)

Published in the August 2003 Issue (vol. 50, no. 4) of IEEE Transactions on Nuclear Science

[24] “ATLAS Level-1 Muon Barrel Trigger robustness study at X5 test facility"

Proceedings of the 9th Pisa Meeting on Advanced Detectors, Elba, Italy, 25-31 May 2003

(F.Pastore)

Published on Nuclear Instruments \& Methods, A 518 (2004) 529-531

[25] "The Design of the Coincidence Matrix ASIC of the ATLAS Barrel Level-1 Muon Trigger"

Proceedings of the 8th Workshop on Electronics for LHC Experiments, Colmar, France, 9-13 Sep 2002 (R.Vari)

[26] "Prototype Slice of the Level-1 Muon Trigger in the Barrel Region of the ATLAS Experiment"

Proceedings of the 7th Workshop on Electronics for LHC Experiments, Stockholm, Sweden, 10-14 Sep 2001 (R.Vari)

[27] "The Sector Logic demonstrator of the Level-1 Muon Barrel Trigger of the ATLAS Experiment"

Proceedings of the 7th Workshop on Electronics for LHC Experiments, Stockholm, Sweden, 10-14 Sep 2001 (A.Salamon)

[28] "Radiation test and application of FPGAs in the ATLAS Level 1 Trigger"

Proceedings of the 7th Workshop on Electronics for LHC Experiments, Stockholm, Sweden, 10-14 Sep 2001 (V.Bocci)

[29] "Radiation tolerance evaluation of the ATLAS RPC coincidence matrix submicron 
technology"

Proceedings of the 6th Workshop on Electronics for LHC Experiments, Cracow, Poland, 11-15 Sep 2000 (R.Vari)

[30] "Irradiation tests of the on-detector components"

E.Gennari et al. presented during the PAD OR, splitter box, optical link PRR, CERN, 30 May 2003

[31] "Test Beam results and integration of the ATLAS Level-1 Muon Barrel Trigger" M. Bianco et al.

Proceedings of the 2004 IEEE Nuclear Science Symposium, Portland.

[32] "Certification of the ATLAS Muon Barrel Chambers"

A. Di Girolamo et al.

Proceedings of the 2006 IEEE Nuclear Science Symposium, Portland.

[33] "The ATLAS LVL1 Barrel Muon Trigger Commissioning with Cosmic Rays"

A. Di Girolamo et al.

Proceedings of 2006 Nuclear Science Symposium and Medical Imaging Conference, San Diego, California, October 29, 2006 (S.Veneziano)

[34] "The ATLAS Barrel Level-1 Muon Trigger Calibration"

A. Di Girolamo et al.

Published as ATLAS Note ATL-DAQ-CONF-2007-020

Proceedings of the 12th Workshop on Electronics for LHC Experiments, Valencia Spain, 25-29

Sept. 2006 (R.Vari)

[35] "The initialization and control of the ATLAS Level-1 Muon Barrel Trigger System"

A. Di Girolamo et al.

Proceedings of the XV International Conference on Computing in High Energy and Nuclear Physics, Mumbai, India, Feb. 13-17, 2006 (S.Veneziano)

[36] "The Read-out Driver for the RPC of the ATLAS Muon Spectrometer"

A. Aloisio et al.

Proceedings of the 2007 IEEE Nuclear Science Symposium, Portland.

[37] "RPC cosmic ray test in the ATLAS experiment"

A. Aloisio et al.

Published in Nuclear Instruments and methods A 581 (2007) 213-216.

Proceedings of the 11th Vienna Conference on Instrumentation (VCI), Vienna, Austria, Feb. 2007 (G.Chiodini) 